\title{
An open digital environment to support business ecosystems
}

\author{
A. Razavi, S. Moschoyiannis, Member, IEEE, and P. Krause, FIMA
}

\begin{abstract}
We present a Peer-to-Peer network design which aims to support business activities conducted through a network of collaborations that generate value in different, mutually beneficial, ways for the participating organisations. The temporary virtual networks formed by long-term business transactions that involve the execution of multiple services from different providers are used as the building block of the underlying scale-free business network. We show how these local interactions, which are not governed by a single organisation, give rise to a fully distributed $P 2 P$ architecture that reflects the dynamics of business activities. The design is based on dynamically formed permanent clusters of nodes, the so-called Virtual Super Peers (VSPs), and this results in a topology that is highly resilient to certain types of failure (and attacks). Furthermore, the proposed P2P architecture is capable of reconfiguring itself to adapt to the usage that is being made of it and respond to global failures of conceptual hubs. This fosters an environment where business communities can evolve to meet emerging business opportunities and achieve sustainable growth within a digital ecosystem.
\end{abstract}

Index Terms - value networks, long-running transactions, lock schemes, concurrency, partial results, scale-free networks, connectivity, Virtual Super Peers, reconfiguration, digital ecosystems

\section{INTRODUCTION}

A digital ecosystem is a self-organising digital infrastructure aimed at creating a digital environment for networked organisations that supports cooperation, knowledge sharing, the development of open and adaptive technologies and evolutionary business models. In the Knowledge Economy, value is no longer created exclusively through the traditional value chain; latest business thinking appreciates the pivotal role of intangibles along with a thorough understanding of the network dynamics [1]. Interest in the value network, rather than the value chain, has increased and opened up the space for considering concepts observed in living organisms that appear in studies of biodiversity. In this thinking, enterprises and business relationships are modelled as living, autopoietic networks.

Our work is directed at supporting this paradigm change in business modelling from linear value chains to value networks. There are two keys aspects to this shift in

Department of Computing, Faculty of Engineering and Physical Sciences, University of Surrey, Guildford, Surrey, GU2 7XH, United Kingdom understanding of the dynamics of business processes. Firstly, business typically does not operate in a linear, assembly line like, flow of material enhancements to tangible assets. Instead, it is conducted through a network of collaborations that generate value in different, mutually beneficial, ways. Secondly, both tangible, and intangible assets generate "value". Access to an e-commerce customer base is an example of a tangible asset while data about the behaviour of the customer base can be understood as an intangible asset.

Although there are a number of larger organisations that have facilitated significant transformations by analysing their business from a value network perspective, this more "ecological" approach to business has been little exploited by the sector that stands to benefit the most from this: Small to Medium Enterprises (SMEs). Part of the reason for this is that such analyses and, more importantly, the computational infrastructure that supports the business transformation, is centralised and thus controlled and set by a single or a handful of (typically large) organisations. This provides a significant barrier to adoption by SMEs, as that central point of control will provide an unacceptable degree of dominance over the participating SMEs.

In contrast, we aim to push down this barrier to adoption by providing a computational infrastructure that supports open collaborations of SMEs that enables a business community to evolve to meet emerging business opportunities, without violating their local autonomy - they only reveal what they choose to reveal to other participants, hence maintaining control over both tangible and intangible assets and their own business strategy.

Interest in the Digital Ecosystems research (e.g. see [17], [33]) comes from a variety of disciplines, from computer, to social, to natural sciences and this manifests itself in the manifold potential applications that range from business transactions and virtual organisations to e-learning platforms and virtual universities.. A number of defining features from a technological and socio-economic perspective underline the Digital Economy initiative. Our interest is in providing support for long-running business transactions and the underlying distributed network architectures that enable these business interactions.

Our approach advocates the local coordination of distributed transactions which involve the deployment of different services from various participating organisations. In addition to the absence of a central point of command and control, and consequently of single point of failure, our 
transaction model builds on fundamental principles of ServiceOriented Computing (SOC) [32], [37], [20]. We address multiservice transactions at the deployment level and show how these can be coordinated in loosely-coupled manner.

A digital ecosystem for business can be largely understood as a digital infrastructure that enables SMEs to combine services and perform core business activities or offer integrated solutions. By its very concept, or at least to encourage participation and facilitate sustainability, the network interconnecting the various participating entities needs to be steered away from centralised solutions, which inevitable create dependency on the provider of the centralised infrastructural unit, and towards fully distributed solutions where each participant makes part of its unused computational resources available for infrastructure maintenance.

It transpires that peer-to-peer (P2P) solutions lend themselves naturally to digital ecosystem architectures. More specifically, we are interested in providing a fully distributed $\mathrm{P} 2 \mathrm{P}$ network that can support long-running transactions between the networked organisations. A digital ecosystem is rather volatile, in terms of the characteristics of the participants (e.g. SMEs as opposed to large enterprises) and the interactions between them. This means that in addition to being fully distributed, the underlying P2P network should be highly dynamic in that it's topology can adapt to the actual usage made of the network. Moreover, a dynamic network topology is better suited to cope with various kinds of failure, which are likely in a highly volatile environment, especially when there is no central point of command and control.

In previous work [19], [4] we have described a model for coordinating distributed long-running transactions and have considered a P2P network design that can support them without creating dependency on any mediator in the network.

In this paper, we are concerned with the interrelationship between the local interactions, in terms of transactions, and the network structures that they give rise to. In particular, we show how these structures can be exploited in creating a P2P network of interconnected smaller networks which are the result of long-running transactions that correspond to automated (B2B) business activities. We show how the design concept of Virtual Super Peers (VSPs) results in P2P network with a dynamic topology which reflects the local interactions between the participants of a digital ecosystem. The resulting framework provides an environment which is highly resilient to certain types of failure both at the transactional level and the network level.

The emergence of the overall network can be achieved when there is certain confidence that the transactions can be deployed correctly. For this purpose, we describe a lock scheme to cover data-orientation in multi-service transactions and show the correctness of the transactional model in this respect. In contrast to conventional transaction models which rely on isolation theorems [23] and two-phase locking (2PL) [26], we use the wormhole theorems for avoiding cycles in each local coordinator of a transaction participant. This has the advantage that a centralised synchroniser as a coordinator is no longer needed for 2PL and transaction commit.
The remainder of this paper is structured as follows. In Section II we outline the basic concepts from digital business ecosystems that are used in the sequel. Section III is concerned with formal proof of correctness of long-running transactions, specifically with respect to consistency of their results. In Section IV we explain the interrelationship between transaction networks and the associated lock schemes and log structures, and show how these can be used to deal with failures during execution. In Section V we describe the overall network design for a digital ecosystem and introduce the concept of VSPs. Implementation experiences so far are discussed in Section VI along with current and possible future plans for extensions. The paper finishes in Section VII with some concluding remarks and possible future directions.

\section{BUSINESS TRANSACTIONS IN A DIGITAL ECOSYSTEM}

The purpose of a business network is to enable networked organisations to engage in distributed business transactions [4] that realise their core business activities. If such a network is to support B2B interactions between SMEs it should be fully distributed (no central point of control for performing a transaction or network operations) and should also offer a consistent model for performing transactions. This means it should be highly resistant to failures - a steady environment where business transactions can be executed.

Each business transaction is the result of peer to peer interactions, between several nodes (SMEs) of this network for reaching a specific target. These nodes are called participants, their logical components for involving in a transaction are their services. The context of a transaction clarify the orchestrations of services, the locality of services clarify the necessary interactions between participants for satisfying the business logic of the transaction. Therefore we are dealing with a service oriented environment, which includes several overlapping networks of transactions.

\section{A. Service-oriented environment and the deployment layer}

In simple terms Service-Oriented Computing (SOC) [31], [32], [20] aims to enable applications from different providers to be offered as services that can be used, composed, and coordinated in a loosely coupled manner. In this paradigm, services are fundamental elements for developing solutions. They are platform-agnostic computational elements that support rapid, low-cost composition of distributed applications. Services perform functions, which can be anything from simple requests to complicated business processes. The actual architectural approach of SOC is called SOA and is particularly applicable when multiple applications running on varied technologies and platforms need to communicate with each other. In this way, enterprises can mix and match services to perform business transactions with minimal programming effort. SOA is a way of reorganizing software applications and support infrastructure into an 
interconnected set of services, each accessible through standard interfaces and messaging protocols. In this way, enterprises suppose to use composition of services to perform business transactions with minimal programming effort and provide a consistent environment.

However, service composition has several distinct characteristics which distinguish it from classical workflow integration, conventional transactional implementation and software component integration. As SOA is a loosely coupled environment, service composition relies on parameters for invocation of a service (also called access interfaces), rather than working with the local state of execution of the respective services. Additional complexity results from the fact that a variety of services, from different platforms, need to pass their results in a loosely coupled manner.

At the same time, the complexity of different types of service composition requires a consistency model at the deployment level. Meanwhile since the locality of services is distributed (in different participants / service providers or SMEs), the quality of service composition in a business transaction depends on the degree of connectivity that the underlying network exhibits.

\section{1) Loosely coupling and service realization}

In SOA services are considered as atomic units whose local structure, or run-time state, cannot be forced to be made visible or explored by other parts of the architecture. The use loosely-coupled services is a basic premise in the serviceoriented computing paradigm, which distinguishes between two broad aspects of services [20], [32] as shown in Fig. 1: service deployment, which is subjected to our transactional service composition, as opposed to service realization.

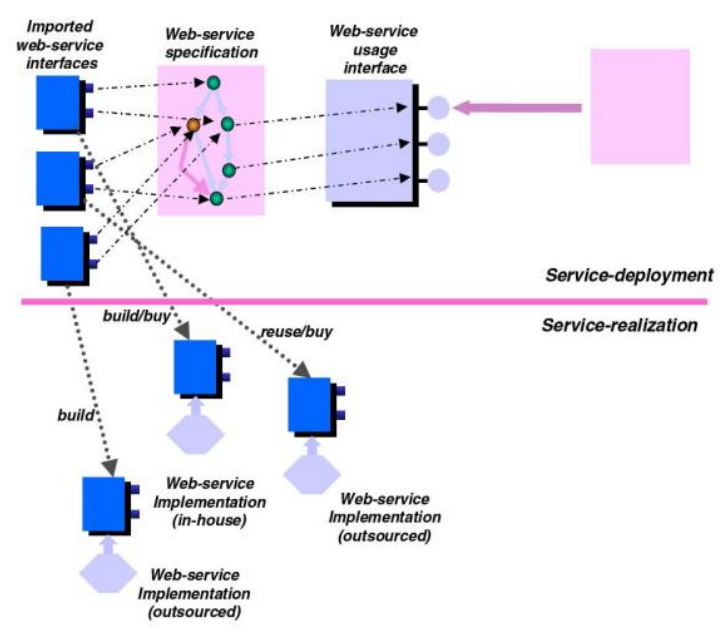

Fig. 1 Realization and deployment levels in SOC [32]

The service realization strategy involves choosing from an increasing diversity of different options for services, which may be mixed in various combinations. Our approach abstracts away from the service realisation level but at the logical level, what we consider is 'there is a business function implemented in software somehow and this is the interface to it' [32].

A service in SOA is designed in such a way that it can be invoked by various service clients and is logically decoupled from any service caller (loose coupling). This means there are no assumptions of any kind in the service as to what kind of service consumer is using it and for what purpose and in what context. In turn, the service callers are coupled with the service in as much as they know what the services are, how they can be used, and what they can accomplish.

Therefore, at the service deployment level the interfaces of the services will be used to compose different services and accomplish the required behaviours and results in terms of business transactions.

2) Service compositions and long-running transactions

Based on the specification advocated in [31],[20],[32] service composition can be considered along the following dimensions: data, process, security, protocol. In this paper we are concerned with providing $\mathrm{P} 2 \mathrm{P}$ network support for distributed transactions and hence we will be concerned with the aspects of data and process composition. In general, security and protocol compositions are usually addressed on top of the transactional layer.

In particular, process-oriented service composition is concerned with the following aspects:

Order: indicates whether the composition of services is serial or parallel.

Dependency: indicates whether there is any data or function dependency among the composed services.

Alternative service execution: indicates whether there is any alternative service in the service composition that can be invoked - alternative services can be tried in either a sequential or a parallel manner.

Following [31] these aspects can be seen within different types of service composition as follows.

Data-oriented service composition: The data generated at the service realisation level are released in terms of different data-objects. In this service composition, these data can be shared and manipulated between participants of a single transaction or, where partial results are concerned, be shared by participants of other transactions.

Sequential process-oriented service composition: This type of service composition invokes services sequentially. The execution of a component service is dependent on its previous service. These sequential dependencies can be based on commitment in which case we talk about Sequential with commitment dependency (SCD) where one cannot begin unless the previous service commits, or data in which case we talk about Sequential with data dependency (SDD) where one service relies on other service's outputs as its inputs.

Parallel process-oriented service composition: In this service composition, all the services can be executed in parallel. There may be data dependencies between them in which case we talk about Parallel with data dependency (PDD) or there may be differences in how and when the services can be committed (depending on some condition) in which case we talk about Parallel with commit dependency 
(PCD). When there are no dependencies between the parallel services we talk about Parallel without dependency (PND).

Alternative service composition: This type of service composition indicates that there are alternative services to be deployed and one of them is necessary. They are categorised to two different types: Sequential alternative composition (SAt) where there is an ordering for deployment of these services, and Parallel alternative composition (PAt), where there is no ordering (preference) between them and deployment of either service can satisfy the composition.

Generally, one or more service compositions can satisfy the user request. It can be seen that due to order and data in service composition, there can be increased complexity in composing services especially when transactions require a number of different services from different networked organisations. This means that there is a need for a context and data consistency model (at the deployment level) which can provide the correctness of the results.

A digital ecosystem for business can be modelled as a network of business transactions between the various participating organisations (e.g., SMEs). Fig. 2 shows the network structure resulting from a number of service compositions which need to be deployed for performing business transactions. Meanwhile in terms of deployment of services and sharing their data (data dependency aspect of service compositions), the peer-to-peer interactions between the participants of each transaction should be supported by a reliable infrastructure which reduces the possibility of failure and provides effective recoverability for the transaction taking place.

\section{B. Network of business transactions}

The aggregations of the business activities taking place between the different partners create several virtual business networks. When these business activities are conducted by means of long-term transactions which involve the execution of services from different service providers, these result in the creation of temporary networks interconnecting the participating organisations. These are typically separate disconnected networks resulting from transactions between participants, but overlaps may exist due to some participants being involved in more than one transaction in the same or different business domain of the digital ecosystem.

Fig. 2 shows the conceptual unstructured network of a digital ecosystem. It can be seen in th4 figure that transactions can have overlapping sets of participants.

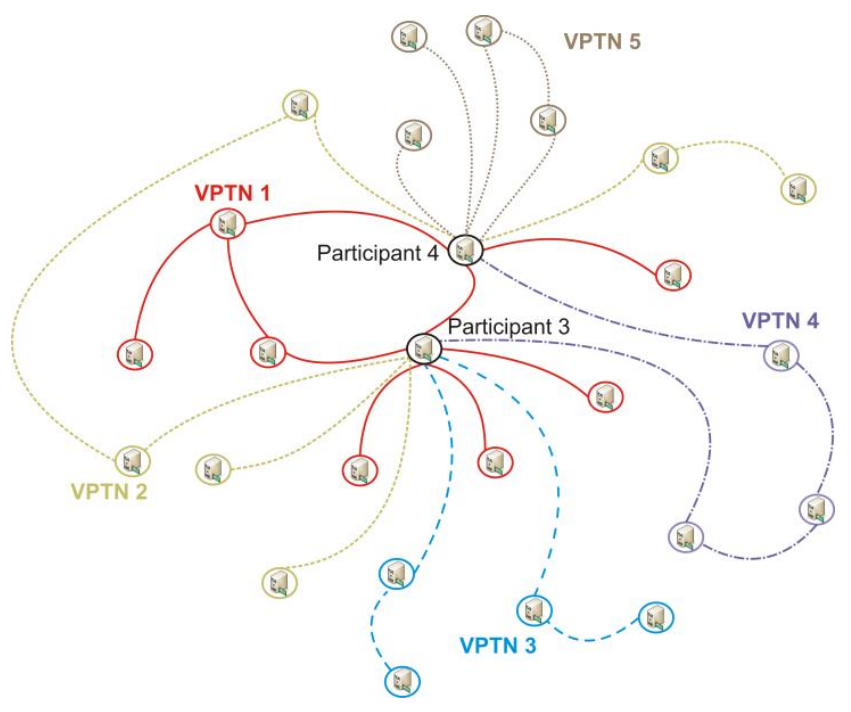

Fig. 2 A Digital Ecosystem

In a digital ecosystem, each transaction and its participants, during the execution of the transaction, creates a temporary network. This network is supposed to achieve a specific goal (transaction goal) and provide some results. During the execution of the corresponding transaction, several services will be deployed. Based on the transaction logic, the underlying services will be deployed in a specific order and their results will be combined (according to the specific service compositions used in the transaction). All of these will be private to the participants of the transaction in question. The resulting network is called 'Virtual Private Transaction Network' or in short form 'VPTN'. Fig 2 shows a digital ecosystem which includes five VPTNs.

The idea is to re-use the (possibly disconnected) VPTNs in providing a connected network for conducting business activities. In a digital business ecosystem, the main motivation of the participants (SMEs) is their business activities (transactions), and thus these sub-networks (VPTNs) are the major part of the system and their success or failure is a major factor for the usability of the ecosystem. For this reason, and before describing the specifics of our approach towards the $\mathrm{P} 2 \mathrm{P}$ architecture that supports distributed business transactions in Section IV, we describe our distributed transaction model focusing on the aspects that are relevant to the underlying supporting network.

\section{Challenges}

As each VPTN is formed by the execution of a transaction amongst the various participants, the very existence of the VPTN relies on the success of the transaction which in turn relies largely on the accessibility of its participants' services. The general term 'Transaction' has been introduced by Gray [22], [23] and is defined by the four properties contained in the ACID acronym. A transaction that is started when a system is in a consistent state may make the state temporarily inconsistent, but it must terminate by producing a new 
consistent state - Consistency is the $\mathrm{C}$ in ACID. This temporary inconsistency may not affect other concurrent transactions. This maintains the illusion that each transaction runs in Isolation - the I in ACID. This means that the inputs and consequent behaviour of some part of the transaction processing system may be inconsistent, even though each transaction executed in isolation would be correct. It follows that concurrent execution should not cause application programs to malfunction, which is the first law of concurrency control. Equally, if some operation within a transaction should fail, it should automatically undo all previous actions and return to the original consistent state. This property is Atomicity, the A in ACID. Also, none of the updates or messages of committed transactions should be lost Durability is the D in ACID.

In a transactional environment, consistency should be preserved even when a failure is encountered and the aborted transactions can be rerun. This main principle applies equally to long-running business transactions, whose execution is long-term in nature - anything from minutes to hours to days and whose specification involves the deployment of a number of services from different service providers. For example, a transaction can include several service compositions and after deploying the data at the deployment level, the data can be accessed several times during the course of execution of the transaction and until it commits. Furthermore, the data may be required to be shared with another transaction before the first one commits, in what is pften referred to as a case of partial results. The conventional ACID properties for transactions may not be applicable in such cases because the transaction life-cycle usually can be longer than that of conventional transactions. Also, partial results can violate the conventional isolation expectation of ACID transactions [18], [23], [28]. It transpires that adhering to ACID properties may be rather restrictive in a business context as a number of B2B scenarios would for instance require the realisation of partial results.

In what follows, we briefly outline current approaches towards relaxing ACID properties and highlight the potential weaknesses with respect to three main criteria: concurrency control (this can cover Consistency and Isolation), compensation and recovery (this covers Atomicity) and replication (for satisfying Durability).

A consortium of companies came together under the umbrella of the Organization for Advance Structured Information Systems (OASIS) and developed the Business Transaction Protocol (BTP), which was aimed at B2B transactions in loosely-coupled domains such as Web services [21]. At the same time, others in the industry released other specifications: Web Services Coordination (WS-Coordination) and Web Services Transactions (WS-AtomicTransactions and WS-BusinessActivity) [27], [29]. Recently, Choreology Ltd. has started to make a joint protocol which tries to cover both models and this effort has highlighted the caveats of each as mentioned in [12], [13], [30].

In what follows we discuss certain important aspects in transactional models and highlight potential pitfalls of current transaction frameworks.

\section{1) Concurrency Control}

For providing a consistent environment, during concurrent actions (service deployment and compositions), WS-* (WSAtomicTransactions and WS-BusinessActivity) and BTP, are using the two-phase commit (2PC) protocol, which requires synchronisation for the phases. This is applied through a centralised coordination framework, based on WSCoordination [24]. Fig. 3 shows a simple example of the use of WS-Coordination for executing a transaction where the Initiator creates a coordination context and the Participants, based on their registered services, deploy their respective services. The synchronisation for concurrency control is done in a centralised manner. This causes a single point of failure as well as a single dependency on the provider(s) of the centralised coordinator framework.

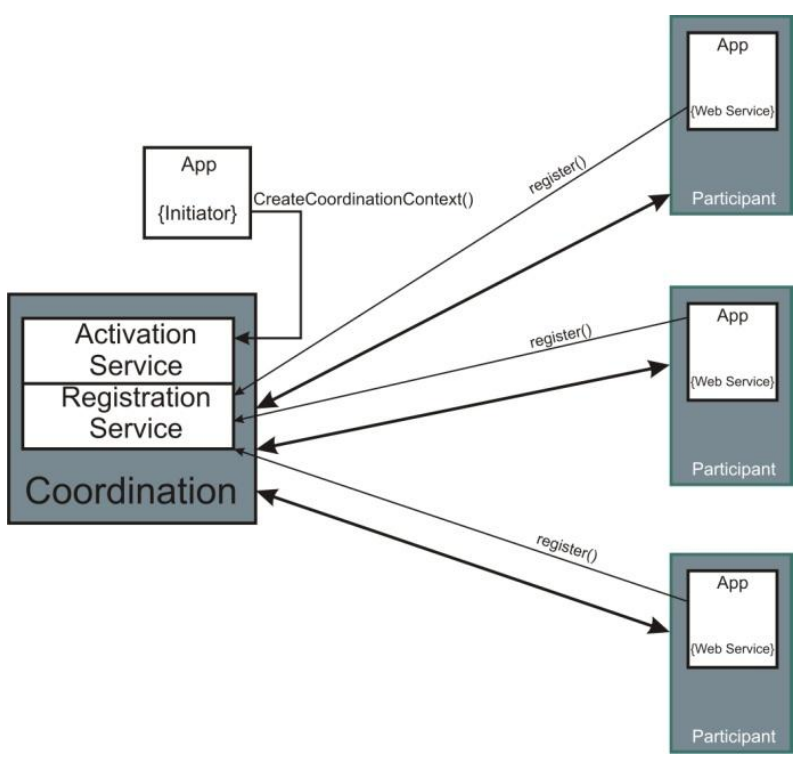

Fig. 3 Centralised coordination

In addition, a more careful study of this coordination framework, such as that reported in [13], shows it to suffer from some critical decisions about the internal build-up of the communicating parties - a view also supported in [12]. The Coordinator and Initiator roles are tightly-coupled and the Participant contains both business and transaction logic. These presumptions are against the primary requirements of SOA, particularly loose-coupling of services and local autonomy, and thus are not suitable for a digital business ecosystem, especially when SMEs are involved. This is because smaller organisations tend to be more sensitive in revealing their local design and implementation precisely because this is often where their model lies [14], [20].

\section{2) Compensation and Recovery}

In a highly dynamic environment of multiple services from different service providers there need to be procedures in place that allow cope with failure. When a failure occurs before a long-running transaction terminates its execution (before 
transaction commit) there is a serious risk of inconsistency due to the fact that released results of the transaction have not been finalised. 'Recovery' is the procedure for addressing this problem. The mechanism, which has been used by WS-* and BTP is compensation. As the coordination protocol for longrunning transactions only uses BACC (Business Activity with Coordinator Completion) protocol, in terms of success or failure, it is the Coordinator who can send the completed message after which the transaction transitions to the second phase (commit or abort) of the 2PC protocol.

Behavioural patterns such as "validate-do" and "provisional-final" [12], [2], [30] are not supported while the "do-compensate" pattern, which is supported, results in a violation of local autonomy, since access to the service realisation level is required.

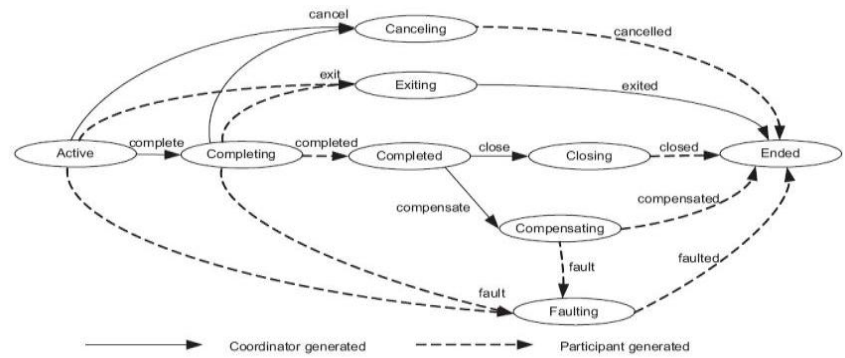

Fig. 4 BACC protocol

As a result of only supporting BACC and hence applying the "do-compensate" behaviour pattern, when a fault occurs, the transaction transitions to 'Faulting' state (see BACC in Fig. 4), where only the Participant is involved. Since the control is with the Coordinator in this case (BACC), the Coordinator needs to have visibility of the Participant's states. Notice that after 'Completed', the 'Closing' and 'Compensating' states are controlled by the Coordinator. This has the implication of the Coordinator needing all details of the Participant to perform the compensating procedures. This limitation results in breaking the autonomy of the local platform as it forces to prescribe the internal behaviour of the realisation level of services. Prescribing internal behaviour at the realisation level raises a barrier for SMEs as it inevitably leads to their tight-coupling with the Coordinator.

\section{3) Replication and durability}

As synchronization is synchronised, the consistency log and recoverability (compensation) information, has stored in centralised coordinator, and as there is not any infrastructure for checking other participants stability, and issuing the privacy of each transaction, the replication and archive of data, are done by a centralised backup in the coordinator, (even when the archiving the information of other participants' transaction can violate the privacy and their local autonomy, this seems like the only option in the current protocols).

It can be seen that there are a number of challenging aspects in providing support for long-running transactions so that the corresponding VPTNs can be used faithfully in determining the connected network supporting a digital ecosystem. In the next section, we describe a distributed transaction model that is designed to address such aspects and paves the way for using the emerging VPTNs as the main building block for the underlying P2P network for digital business ecosystems.

\section{CORRECTNESS OF DISTRIBUTED VPTNS}

As discussed in the previous section, current protocols in transaction frameworks targeted at supporting business activities between networked organisations provide a centralised solution, which not only violates the primary concept of SOC (loose-coupling) but also does not cover all aspects of their business activities. This creates tight dependencies which are susceptible to the risk that comes with a single point of failure in the framework. In contrast, our purposed model, from the very beginning and early prototypes, advocates a fully distributed solution and relies on the P2P interactions between the platforms (here, participants) [30], [14].

As the kernel of each platform, we have designed a software agent which is responsible for coordinating the participant's business activities (transactions). This local agent also archives the information related to these activities (corresponding VPTNs) and improves the general connectivity of the network (its digital ecosystem), and in doing so it contributes to the so-called network growth [14]. This is an important aspect when it comes to sustainability, especially in a fully distributed solution. This leads up to the main definition of a digital ecosystem (recall section II) which is represented in Fig 2 highlighting the fact that there is no centralised point of command and control in a digital ecosystem.

Fig. 5 shows the structure of the local software agent of each participant. The 'local coordinator' component coordinates the service requests to and from the local platform. In other words, it deploys services of the platform, coordinates the transactions and archives their information in the 'local service repository'. In this way, all participants of a transaction will keep the archived information of the transaction. The 'local web service informer' component updates any changes of local services in the 'local service repository' and relevant participants can be notified of the changes through the 'web service promoter'. The links to other participants will be kept in the 'global service repository'. Note that at this stage, participants of different VPTNs are connected to each other (in [14] this is called the birth stage of the underlying network). For reducing the possibility of failures (discussed in further detail in Section V) and increasing the network stability (Section VI), the network connectivity, i.e. the number of links to other participants, may change. These changes will be done by two components; the 'web service information investor', for updating new links to the global repository and the 'web service promoter', for promoting new links to other participants (in [14] this is referred to as the growth stage). 


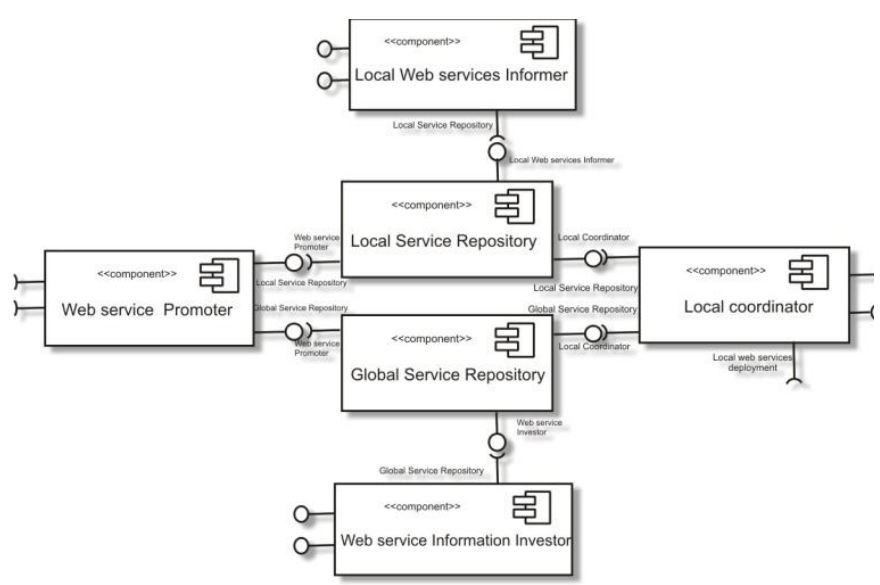

Fig. 5 The structure of Participant's Software Agent

\section{A. Transaction context}

A transaction can be modelled as a nested structure of subtransactions [26]. These can be composite based on data or order (each sub-transaction acts like a service in the service composition framework discussion (Section II, A, 2) and more details can be found in [31]). In that way, the lowest part will be services in each participant (the local coordinator shown in Fig. 5, can deploy them through one of its interfaces). Fig 6 shows such a structure where we have five services, which have been combined by different service compositions. The notation symbols used here are based on [28] and 'Seq' is for Sequential, 'Par' for Parallel and 'Alt' for alternative service compositions.

This is called the transaction context and it is sued to clarify the semantics of a transaction. We have described this semantics in [3] and also advocated a methodology for optimising this, in terms of behavioural properties of the interactions involved, in our previous works [19]. Meanwhile the details of this context, including the $\mathrm{xml}$ presentation of this example (given in Fig. 6), the xml schema for creating the tree and other infrastructural schemas relevant to this paper can be downloaded from [34]. Furthermore, the Java parser for the model components is also available through the same link.

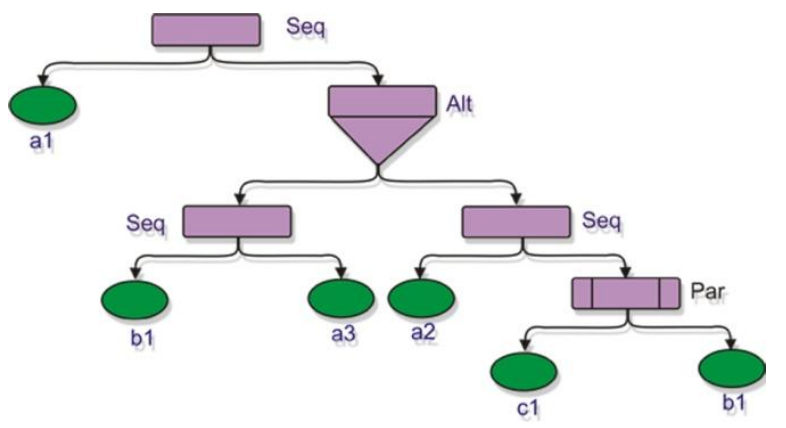

Fig. 6 Transaction Tree

As we have seen in Section II, in terms of service composition, it is possible to have different levels of datadependency between sub-transactions of a transaction - what is referred to as long-running transaction [26], [20]. In terms of concurrent execution of sub-transactions of a transaction, this may cause data-inconsistency which is considered as one of the very first requirements of a transaction (recall $\mathrm{I}$ in ACID, which has been described in Section II, C). This is the first challenge for a digital ecosystem, which will be addressed in the rest of this section. The remaining two challenges, Recoverability and Durability will be addressed in Sections IV and $\mathrm{V}$.

As the first step, we look at a transaction (to be precise, a long-running transaction) as a set of sub-transactions, which may use each other's objects (data-items):

$$
T=\left\{S T_{1}, S T_{2}, \cdots, S T_{n}\right\}
$$

\section{1) Concurrency and Isolation theorems}

The classic view of isolation considers the transaction in terms of inputs and outputs [22], [23]. In our approach, this means that sub-transactions have read (input) and write (output) operations. Write operations are understood as operations that affect the state of resources. This means it it appropriate to consider read operations as inputs for subtransactions and write operations as their outputs. Then isolation between two sub-transactions can be expressed as:

$$
\boldsymbol{O}_{\boldsymbol{i}} \cap\left(\boldsymbol{I}_{\boldsymbol{j}} \cup \boldsymbol{O}_{\boldsymbol{j}}\right)=\emptyset \text { for all } \boldsymbol{i} \neq \boldsymbol{j} \quad \mathrm{EQ} .1
$$

Let $I_{i}$ be the set of objects read by sub-transaction $S T_{i}$ (its inputs), and $O_{i}$ be the set of objects written upon by the subtransaction $S T_{i}$ (its outputs). Based on EQ.1, the set of subtransactions $\left\{S T_{i}\right\}$, for all $i$, when their outputs are disjoint from one another's inputs and outputs, they can run in parallel with no concurrency anomalies. Hence, by applying EQ.1 any sub-transaction scheduler can work.

Conventionally, for applying EQ.1 each sub-transaction should declare its input-output set and then a scheduler is able to compare the new sub-transaction's need to all running subtransactions and in case of a conflict, the initiation of the new sub-transaction would be delayed until the conflicting subtransactions complete. This approach is called 'Static allocation'. It has been argued however that the computing complexity of analysing the inputs and outputs before running transactions can cause a bottleneck on scalability [23], [25].

The 'Dynamic allocation' scheme has been introduced as an alternative approach. Under the prism of dynamic allocations sub-transactions can be viewed as sequences of operations on deployed objects. A particular object is subject to one operation at a time. Each operation of a sub-transaction is either read (using the object as the input for service deployment/composition) or write (over-writing/updating the object as the output of a service deployment/composition).

Objects go through a sequence of versions as they are updated by write operations. In contrast, read operations do not change the object version. If a sub-transaction reads an object, the sub-transaction depends on that object version. If the sub-transaction writes an object, the resulting object version depends on that sub-transaction. When a sub- 
transaction aborts and goes through the undo logic, all its write operations must be undone. This results in the object getting a new version, thus the 'undo' looks like an ordinary new update.

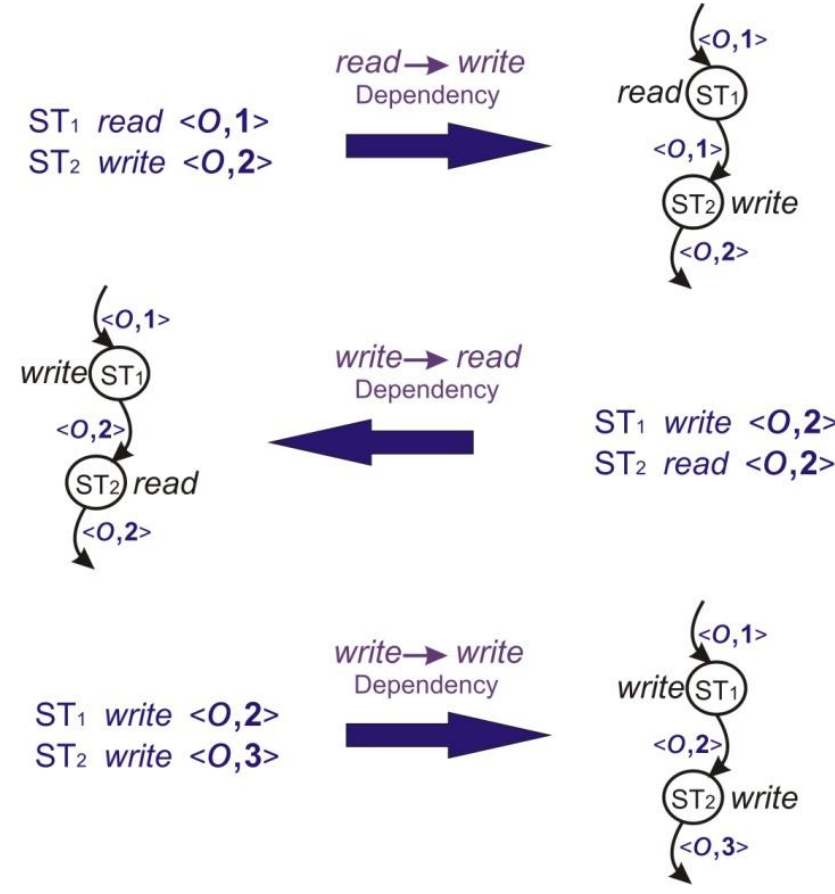

Fig. 7 Dependency Graph

Theoretically, a dependency graph can be read as a time sequence. In Figure 7, an edge from sub-transaction $\mathrm{ST}_{1}$ to $\mathrm{ST}_{2}$ indicates that $\mathrm{ST}_{1}$ accesses an object later accessed by $\mathrm{ST}_{2}$, and at least one of these access operations created a new version. In that sense, $\mathrm{ST}_{1}$ ran before $\mathrm{ST}_{2}$. In a purely sequential execution of the transaction - running $\mathrm{ST}_{1}$ to completion, and only then running $\mathrm{ST}_{2}$ to completion - all dependency arrows will point from $\mathrm{ST}_{1}$ to $\mathrm{ST}_{2}$. However, as the execution of the transaction depends on the semantics (transaction context), there can be different composition types; in parallel execution, the dependency arrows can form an arbitrary graph. This brings about the issue of cyclic dependencies, which should be avoided as they can give rise to concurrency anomalies.

The main conclusion of applying the transactional properties is that any dependency graph without cycles implies an isolated execution of the corresponding sub-transactions. So if the dependency graph does have cycles, then the subtransactions were not executed in isolation. If the dependency graph has no cycles, then the sub-transactions can be topologically sorted to make an equivalent execution history in which each sub-transaction can be ran serially, one completing before the next began. This result in conventional transactions has been given in [23], [25] and implies that each sub-transaction ran in isolation, as if there was no concurrency; it also implies that there were no concurrency anomalies.

It is not difficult to see that violation of the isolation property is related to the various dependency cycles. Similarly to conventional transactions, cyclic dependencies in long- running transactions are categorised into three generic forms:

Lost updates: The first sub-transaction's write (deploying the data) is overwritten by the second sub-transaction which uses write based on the initial value of the object.

In Figure 8, we show the conflict of these writes. The subtransaction $\mathrm{ST}_{2}$ tries to update the object $o$, based on the previous version of the object (denoted by 1 in the figure), while sub-transaction $\mathrm{ST}_{1}$ is updating the object based on the same object version (1). This means that $\mathrm{ST}_{2}$ may update the object without considering the sub-transaction $\mathrm{ST}_{1}$. One of the updates will be overwritten without being taken into account. This is referred to as lost update.

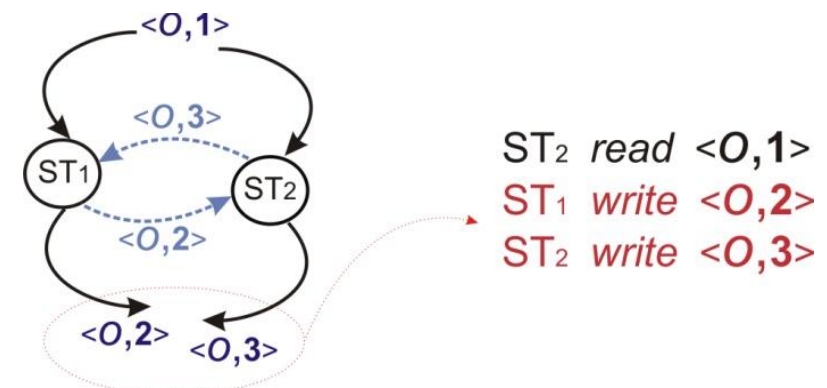

Fig. 8 Lost Update

Since $\mathrm{ST}_{1}$ and $\mathrm{ST}_{2}$ are both using the object $\mathrm{o}$, each write creates a dependency on the other sub-transaction, denoted by the light-blue dashes in Figure 8. These two writes create a cycle of dependencies which results in an inconsistent state for the long-running transaction as a whole.

Dirty read: A sub-transaction reads an object which has been written before by another sub-transaction which also writes to it after the read action. This means the first subtransaction may find inconsistency in the object. This is equivalent with the Phantom Problem in DBMS [22], [18] - in short, this refers to the case where a transaction (T) can read changes made to an object during an ongoing transaction, so the object can be changed further while the transaction $(\mathrm{T})$ is in progress, and as a result the transaction (T) is vulnerable to be accessing inconsistent data.

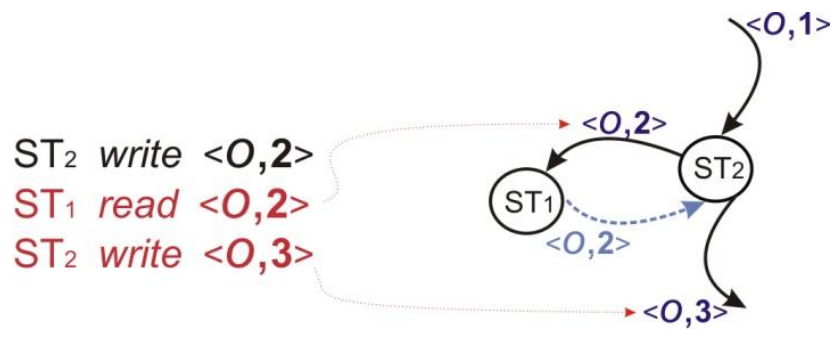

Fig. 9 Dirty Read

Figure 9 shows sub-transaction $S T_{1}$ accessing the object $o$, which is under ongoing changes by sub-transaction $S T_{2}$. Therefore $S T_{1}$ may read inconsistent data from the object $o$, which in this example is the temporary version of the object (version 2), which is supposed to be finalised to version 3 only 
before sub-transaction $S T_{2}$ commits (or aborts). In term of dependencies, when $\mathrm{ST}_{1}$ reads the version 2 of object o it creates a dependency with $\mathrm{ST}_{2}$, denoted by the dashed line in Figure 9, but when $\mathrm{ST}_{2}$ writes on the object the opposite dependency is created, denoted by the solid line. As discussed earlier, it has been shown that this cycle of dependency leads the long-running transaction to an inconsistent state, meaning that in their life-cycle the two sub-transactions are working on two different values for the same object.

Unrepeatable read: In this case, a sub-transaction reads an object twice, once before another sub-transaction's write action and for a second time after the write action (the second sub-transaction may write a new version and commit). This means that a sub-transaction changes the object (write) when another sub-transaction had ongoing access (read) to it and has not yet finalised its access.

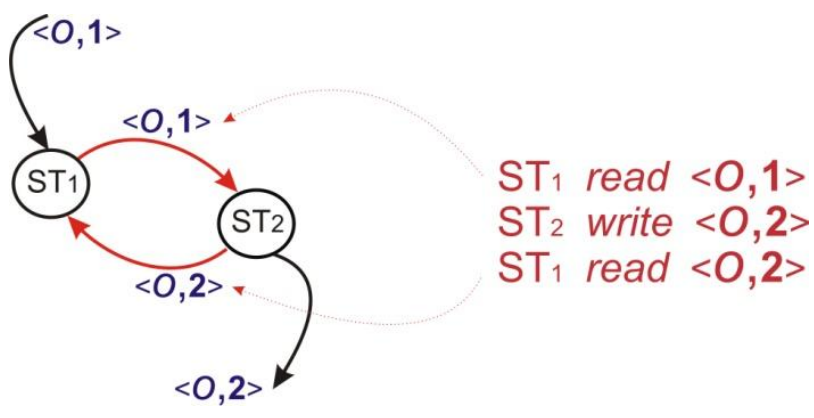

Fig. 10 Unrepeatable read

Figure 10 shows a simple scenario of unrepeatable read where $S T_{1}$ access to an object $o$ and retrieves the version 1 of the object, but if there is a second attempt by $S T_{1}$ the result of read is different because during the execution of $S T_{1}$ the subtransaction $S T_{2}$ had access to the object (write) and changed it to a new version (here version 2). In terms of dependency, write on object $o$ by $\mathrm{ST}_{2}$ after the first read of the object by $\mathrm{ST}_{1}$ creates the read to write dependency, and the next read operation creates a write to read dependency between the same sub-transactions. This results in inconsistency on object $\mathrm{o}$ in the life-cycle of the two sub-transactions which belong to the same long-running transaction.

These simple examples can be extend to more complicated scenarios when considering a few intermediate sub-transaction dependencies where the final dependency returns to the first sub-transaction. These cycles in dependency graphs are called wormholes [23], [25].

The isolation theorems are the classic method for showing the correctness of the transactional environment [23], [26], [25]. The main result of the study in isolation can be summarised as follows: a serial execution of transactions is always correct, when each transaction follows the commit or full rollback of the other one. Therefore, if we show a concurrent execution of transactions is equivalent to a serial execution, we may use the isolation theorem to deduce correctness of the transaction execution.
The conventional isolation theorems use centralised synchronisation for applying the two-phase locking (2PL) scheme [18], [23]. 2PL and lock compatibility guarantee the environment is free of any wormholes [23]. It also shows that an environment without wormholes is isolated, i.e. the transactions' execution is equivalent to a serial execution of them, and thus the system is consistent.

Our approach advocates a fully distributed solution and hence for avoiding the centralised synchronisation we do not use 2PL. The implications of write operations are handled instead using dependency graphs. For any write operation in the semantics of a transaction (in service compositions with data dependency, section II, A, refer to PDD and SDD [31] [20]) it the dependency graphs determines the access rights to the corresponding object. The necessary graphs can be propagated in VPTN. In short, by using the dependency graphs we avoid the wormholes.

Before introducing the dependency graphs, we give the definition of a wormhole, its theorem in our long-running transactional model and the proof for correctness of a wormhole-free long-running transaction.

\section{2) Conventional definitions}

For defining an execution of a long-running transaction we use the standard term history. A history of a long-running transaction is any sequence-preserving merge of the actions of a set of sub-transactions into a single sequence for the set of sub-transactions and is denoted by $H=\left\langle\langle s t, a, o\rangle_{i}\right| i=$ $1, \ldots, n$. Each step of the history $s t, a, o$ is an action $a$ by subtransaction st on object $o$. A history for the set of subtransactions $\left\{S T_{j}\right\}$ is a sequence, each containing transaction $S T_{j}$ as a subsequence and containing nothing else.

In effect, a history lists the order in which actions were successfully completed. Serial histories are onesubtransaction-at-a-time histories. In serial histories as there is no concurrency, there is not any inconsistency and no problem with viewing inconsistent data by other transactions.

As each action in the history changes the version of the object, we need to formalise the versioning definition before defining dependencies between sub-transactions in the history. The version of an object $o$ at step $k$ of a history is an integer and is denoted $V(o, k)$. In the beginning, each object has version zero $(V(o, 0)=0)$. At step $k$ of history $H$, object $o$ has a version equal to the number of writes of that object before this step. Formally, this means:

$$
\begin{gathered}
V(o, k)= \\
\mid\left\{\left\langle s t_{j}, a_{j}, o_{j}\right\rangle \in H \mid j<k \text { and } a_{j}=\text { WRITE and } o_{j}=o\right\} \mid .
\end{gathered}
$$

Note we will use capitalisation for operation from now on in order to make the notation more clear.

Now we are able to define dependency in a history. Each history $H$ for a set of sub-transactions $\left\{S T_{i}\right\}$ defines a threefold dependency relation $\operatorname{DEP}(H)$, defined as follows.

Let $S T 1$ and $S T 2$ be any two distinct sub-transactions, let $o$ be any object, and let $i, j$ be any two steps of $H$ with $i<j$. Suppose step $H[i]$ involves action $a 1$ of $S T 1$ on object $o$, step 
$H[j]$ involves $a 2$ of $S T 2$ on $o$, and suppose there is no write of $o$ by any transaction between these steps (there is no $\left\langle S T^{\prime}, W R I T E, o\right\rangle$ in $\left.H[i+1], \ldots, H[j-1]\right)$. Then $\operatorname{DEP}(H)$ is defined as:

$$
\begin{gathered}
\left\langle S T,\langle o, V(o, j)\rangle, S T^{\prime}\right\rangle \in \operatorname{DEP}(H) \text { if } \\
a 1 \text { is a WRITE and } a 2 \text { is a WRITE } \\
\text { or } \\
a 1 \text { is a WRITE and } a 2 \text { is a READ } \\
\text { or } \\
a 1 \text { is a READ and } a 2 \text { is a WRITE. }
\end{gathered}
$$

This classic definition captures all dependencies (WRITE $\rightarrow$ WRITE, WRITE $\rightarrow$ READ and READ $\rightarrow$ WRITE).

The dependency relation for a history of a long-running transaction defines a directed dependency graph. Subtransactions are the nodes of the graph, and object versions label the edges. This means that if $\left\langle S T,\langle o, j\rangle, S T^{\prime}\right\rangle \in \operatorname{DEP}(H)$, then the graph has an edge from node $S T$ to node $S T$ ' labelled by $\langle o, j\rangle$. It follows that two histories are equivalent, if they have the same dependency relation.

\section{B. Wormhole theorem}

As discussed in the introduction of the paper, in the proof of correctness we will not be applying locking theorems as these rely on two-phase locking (2PL), and as a result require a centralised coordinator for enforcing the necessary synchronisation of each phase. In addition, instead of proving correctness for a transactional environment as a whole, we will be concerned with each individual transaction. We note that any released results (data-items) between transactions will be covered by the conditional commit mechanism, which is a wormhole-free mechanism as will be shown in Section D.

In what follows we will prove correctness of an individual transactions by means of showing it is wormhole-free. The wormhole theorem is a well-known theorem in isolation theorems. Here, we describe how it can be adapted to determine wormhole-free transactions of the kind considered in our approach, i.e. long-running transactions.

Hence, before using the classic theorem and its proof [23] we introduce the equivalent concept and notation in longrunning transactions, and then adapt the proof to long-running transaction.

The dependencies in the history of a long-running transaction can define a time ordering of the sub-transactions. Conventionally this ordering is signified by $<<<_{H}$, (or simply by $<<<$ when the history is clear from context), and it is the transitive closure of $<<<$. It is the smallest relation satisfying the equation $S T<<<_{H} S T^{\prime}$ :

if $\left\langle S T, o, S T^{\prime}\right\rangle \in \operatorname{DEP}[H]$ for some object version $o$, or $\left(S T<<<_{H} S T^{\prime \prime}\right.$ and $\left\langle S T^{\prime \prime}, o, S T^{\prime}\right\rangle \in \operatorname{DEP}[H]$ for some sub-transactions $S T^{\prime \prime}$, and some object $o$ ).

In terms of the dependency graph, we can say that $S T<<<$ $S T^{\prime}$ if there is a path in the dependency graph from sub- transaction $S T$ to sub-transaction $S T^{\prime}$.

The $<<<$ ordering defines the set of all sub-transactions that run before or after $S T$;

$$
\begin{gathered}
\operatorname{BEFORE}(S T)=\left\{S T^{\prime} \mid S T^{\prime}<<<S T\right\} \\
\operatorname{AFTER}(S T)=\left\{S T^{\prime} \mid S T<<<S T^{\prime}\right\}
\end{gathered}
$$

If $S T$ runs fully isolated (i.e., it is the only sub-transaction, or it read and write objects not accessed by any other subtransactions), then its BEFORE and AFTER sets are empty, and it can be scheduled in any way. When a sub-transaction is both after and before another distinct sub-transaction ( $S T$ here), it is called wormhole transaction ( $S T^{\prime}$ here):

\section{$S T^{\prime} \in \operatorname{BEFORE}(S T) \cap \operatorname{AFTER}(S T)$}

It is not hard to see that serial histories do not have wormholes - in a serial history, all the actions of one transaction precede the actions of another, i.e. the first cannot depend on the outputs of the second.

Based on the wormhole theorem, a history is isolated if, and only if, it has no wormhole sub-transactions. On the other hand, the isolated histories have the unique property of having no wormholes. The theorem dictates that a history that is not isolated has at least one wormhole; $S T<<<S T^{\prime}<<<S T$.

In graphical terms we can say that if the dependency graph has a cycle in it, then the history is not equivalent to any serial history because some sub-transaction is both before and after another sub-transaction. Figures 8, 9 and 10 demonstrate simple cases of such cycles. A wormhole in a particular history is a sub-transaction pair in which $S T$ ran before $S T^{\prime}$ which ran before $S T$. A history is said to be isolated if it is equivalent to a serial history.

\section{1) Isolated history has no wormhole}

As the first part of the proof of this concept, the classical testimony of the wormhole theorem has been adopted to longrunning transactions and the proof is an adaptation of that given by Gray in [23].

Theorem. An isolated history has not any wormholes.

This proof is done by contradiction. Suppose that in a longrunning transaction, $H$ is an isolated history of the execution of the set of sub-transactions $\left\{S T_{i} \mid i=1, \ldots, n\right\}$. By definition, $H$ is equivalent to some serial execution history of the same long-running transaction, denoted by $S H$ (obviously for that same set of sub-transactions). Without loss of generality, assume that the sub-transactions are numbered so that $S H=S T_{1}\left\|S T_{2}\right\| \ldots \| S T_{n}$. This means $S H$ is equivalent to starting with the execution of all actions in sub-transaction $S T_{1}$ (followed by) concatenated to the execution of all actions in sub-transaction $S T_{2}$ (followed by) concatenated to ... execution of all actions in sub-transaction $S T_{n}$.

Now suppose that $H$ has a wormhole. We will show that it is impossible for it to be isolated. Having a wormhole means that there is some sequence of sub-transactions $S T, S T^{\prime}, S T^{\prime \prime}, \ldots, S T^{\prime \prime \prime}$ in $H$ such that each is BEFORE the other (i.e., $S T<<<_{H} S T$ ), and the last is BEFORE the first (i.e., $S T^{\prime \prime \prime}<<<_{H} S T$ ). Let $i$ be the minimum sub-transaction 
index such that $S T_{i}$ is in this wormhole, and let $S T_{j}$ be its predecessor in the wormhole (i.e., $S T_{j}<<<_{H} S T_{i}$ ). Since $i$ is minimum, $S T_{j}$ comes completely AFTER $S T_{i}$ in the execution history $S H$, so that $S T_{j}<<<_{S H} S T_{i}$ is impossible (recall that $S H$ is a serial history). But since $H$ and $S H$ are equivalent, $<<<_{H}=<<<_{S H}$; therefore, $S T_{j}<<<_{S H} S T_{i}$ is also impossible. This contradiction proves that if $H$ is isolated, it has no wormholes, or, as we say, is a wormhole-free history.

2) Wormhole-free history is an isolated history

Now for the second part of the theorem we still need to show that a history without wormholes is isolated. In what follows we adapt the classic Wormhole theorem proof [23], [25] but for a long-running wormhole-free history.

If a long-running transaction has $n$ sub-transactions (the number of sub-transactions is $n$ ), then they appear in the history $H$ of the long-running transaction. The induction hypothesis is that any $n$ sub-transactions history $H$ which is wormhole-free is isolated (this means $H$ is equivalent to some serial history $S H$ for that set of sub-transactions).

If $n<2$, then any history of the long-running transaction is a serial history, since only zero or one sub-transaction appears in the history. In addition, we have already seen that any serial history is an isolated history. The basis of the induction, then, is trivially true.

Suppose the induction hypothesis is true for $n-1$ subtransactions, and consider some history $H$ of $n$ subtransactions that has no wormholes. Pick any sub-transaction $S T$, then pick any other sub-transaction $S T^{\prime}$, such that $S T<<$ $<S T^{\prime}$, and continue this construction as long as possible, building the sequence $Q=\left\langle S T, S T^{\prime}, \ldots\right\rangle$. Either $Q$ is infinite, or it is not. If $Q$ is infinite, then some sub-transaction $S T^{\prime \prime}$ must appear in it twice. This, in turn, implies $S T^{\prime \prime}<<<S T^{\prime \prime}$, and hence $S T^{\prime \prime}$ is a wormhole of $H$. But since $H$ has no wormholes, $Q$ cannot be infinite. The last transaction in $Q$, and let us call it $S T^{*}$, has the property $\operatorname{AFTER}\left(T^{*}\right)=\emptyset$, since the sequence cannot be continued past $S T^{*}$.

Consider the history $\mathrm{H}^{\prime}=\left\langle\left\langle s t_{i}, a_{i}, o_{i}\right\rangle \in H \mid s t_{i} \neq S T^{*}\right\rangle$. This says that $H^{\prime}$ is the history $H$ with all the formal actions of transaction $S T^{*}$ removed. By the choice of $S T^{*}$,

$\operatorname{DEP}\left(\boldsymbol{H}^{\prime}\right)=\left\{\left\langle\boldsymbol{S} \boldsymbol{T},\langle\boldsymbol{o}, \boldsymbol{i}\rangle, \boldsymbol{S} \boldsymbol{T}^{\prime}\right\rangle \in \operatorname{DEP}(\boldsymbol{H}) \mid \boldsymbol{S} \boldsymbol{T}^{\prime} \neq \boldsymbol{S} \boldsymbol{T}^{*}\right\} \mathrm{EQ} .2$

$H^{\prime}$ has no wormholes (since $H$ has no wormholes, and $\left.\operatorname{DEP}(H) \supseteq \operatorname{DEP}\left(H^{\prime}\right)\right)$. The induction hypothesis, then, applies to $H^{\prime}$. Hence, $H^{\prime}$ is isolated and has an equivalent serial history $S H^{\prime}=S T_{1}\left\|S T_{2}\right\| \ldots \| S T_{n-1}$ for some numbering of the other sub-transactions.

The serial history $S H=S H^{\prime}\left\|S T_{n}=S T_{1}\right\| S T_{2}\|\ldots\| S T_{n-1} \| S T^{*}$ is equivalent to $H$. To prove this, we need to show that $\operatorname{DEP}(S H)=\operatorname{DEP}(H)$. By construction,

$\operatorname{DEP}(\boldsymbol{S} \boldsymbol{H})=\operatorname{DEP}\left(\boldsymbol{S} \boldsymbol{H}^{\prime} \| \boldsymbol{S} \boldsymbol{T}_{\boldsymbol{n}}\right)=$ $\operatorname{DEP}\left(\boldsymbol{S} \boldsymbol{H}^{\prime}\right) \cup\left\{\left\langle\boldsymbol{S} \boldsymbol{T}^{\prime},\langle\boldsymbol{o}, \boldsymbol{i}\rangle, \boldsymbol{S} \boldsymbol{T}^{*}\right\rangle \in \operatorname{DEP}(\boldsymbol{H})\right\} \quad$ EQ. 3

Also, by definition, we have $\operatorname{DEP}\left(S H^{\prime}\right)=\operatorname{DEP}\left(H^{\prime}\right)$. Using this to substitute equation EQ. 2 into equation EQ. 3 gives:

$$
\operatorname{DEP}(\boldsymbol{S H})=
$$

$$
\begin{gathered}
\left\{\left\langle\boldsymbol{S T},\langle\boldsymbol{o}, \boldsymbol{i}\rangle, \boldsymbol{S \boldsymbol { T } ^ { \prime }}\right\rangle \in \operatorname{DEP}(\boldsymbol{H}) \mid \boldsymbol{S \boldsymbol { T } ^ { \prime }} \neq \boldsymbol{S} \boldsymbol{T}^{*}\right\} \\
\cup\left\{\left\langle\boldsymbol{S T} \boldsymbol{T}^{\prime},\langle\boldsymbol{o}, \boldsymbol{i}\rangle, \boldsymbol{S} \boldsymbol{T}^{*}\right\rangle \in \operatorname{DEP}(\boldsymbol{H})\right\} \\
=\operatorname{DEP}(\boldsymbol{H})
\end{gathered}
$$

Thus, the identity $\operatorname{DEP}(S H)=\operatorname{DEP}(H)$ is established, and the induction step is proven.

The wormhole theorem is the basic result from which all the others follow. It essentially says "cycles are bad". Wormhole is just another name for cycle. The wormhole theorem can be stated in many different ways. One typical statement is called the Serializability Theorem: A history $H$ is isolated (also called a serializable schedule or a consistent schedule) if, and only if, $<<<_{H}$ implies a partial order of the transactions. (Alternatively: if and only if it defines an acyclic graph, or implies a partially ordered set [23]). This is the basis for our log system to create dependency graphs and avoid the bad cycle.

\section{Internal dependency graph}

We have shown that by avoiding wormholes we can release results between sub-transactions of a long-running transaction. In doing so, we use the dependency graph to trace released data items (objects) between each participant. This graph is updated regularly and the cycle (wormhole) can be detected in each step. As this graph captures dependencies between subtransactions of a transaction we call it Internal Dependency Graph (IDG).

For clarifying the access-right, inside of each participant we use a simple lock mechanism which is compatible with the conventional S/X Lock. The only difference with S/X Lock is the UN-Lock mechanism. Since participants are executing a sub-transaction and the result can only be visible in that particular long-running transaction, instead of unlocking the data we introduce an internal lock I-Lock which unlocks the data items in the context of a particular transaction. This means the data item will be available for other subtransactions of the transaction, which are executing in other participants. As mentioned in the beginning of the section, the execution of a transaction will be done by the 'local coordinator' of the participants. Fig 11 shows a simple example. 


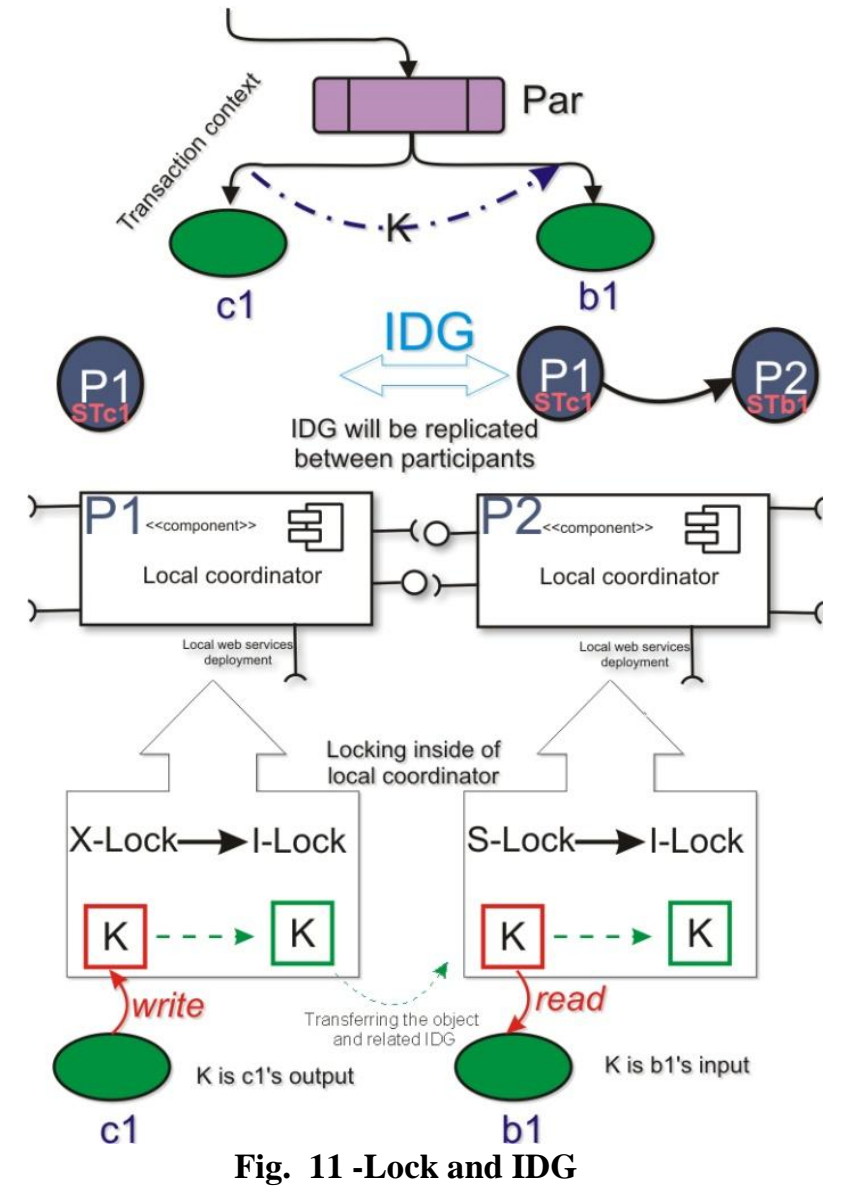

Fig. 11 shows part of a long-running transaction, where a parallel composition between service $\mathrm{c} 1$ and $\mathrm{b} 1$ has a datadependency (recall the composition type PDD in Section II, A). We have assumed that service $\mathrm{cl}$ is a service offered by participant $\mathrm{P} 1$ and service $\mathrm{b} 1$ is of participant $\mathrm{P} 2$. Based on the transaction context described in the beginning of this section (Section III, A), c1 and b1 can be deployed in the context of two sub-transactions (STc1 and STb1). When participant P1, deploys the service $\mathrm{c} 1$ the service creates a data-item $\mathrm{K}$ as the output (notice that P1 uses X-Lock for writing/creating the object) and sub-transaction STc1 commits and releases the result for other sub-transactions. To do this P1 uses I-Lock and pre-request for the object while the IDG can be created for the object. Our example illustrates the case where participant P2 needs the output of c1 (STc1). It can use the result which has been released by I-Lock and if there is a dependency between the two sub-transactions present in the corresponding IDG (STc1 $\ll<\mathrm{STb} 1)$, also shown in the figure, P2 uses S-Lock for reading $\mathrm{K}$ and proceeds to use it as the input in service $\mathrm{b} 1$. Then P2 can again release it by using I-Lock on the data-item. Any subsequent usage of the data-item will be done by checking and updating the IDG. In this way, local coordinators can avoid any cycle (e.g., STc1 $1<<$ STb $1<<<\ldots<<$ STc1).

In further explanation, if there is a dependency between participant P1 and participant P2 inside a transaction, which is indicated in the transaction context (upper part) of Fig. 11, and participant $\mathrm{P} 1$ is operating on a data-item (in terms of subtransaction STc1 in the figure), then this data-item is made available to any other participants (participant $\mathrm{P} 2$ in the figure) once P1 has finished at which point it releases it by using ILock. In the meantime the IDG will be updated and replicated on each participant involved with the given data-item so that all data dependencies are recorded and checked for avoiding any wormholes as discussed in the previous section. In the corresponding transaction context $\mathrm{xml}$ schema, which can be found following [34], this is captured by the complexType element with attributes Originator and Dependent for pairs of participants involved.

\section{External dependency graph}

As the life-cycle of long-running transactions is long, occasionally, releasing results between these transactions before their termination (either commit or rollback) can be valuable for a digital ecosystem of networked organisations [30], [3]. This is both in terms of performance and avoiding unacceptable degradation of performance, but also in covering a wider range of B2B scenarios. However, these partial results across transactions can be costly - in case of abortion of the first long-running transaction we may face cascading abortion [30], [14] of a number of other transactions. This is why they should be used when it is necessary and there is possibility for forward recovery in case of abortion of the first transaction this is further discussed in the next section. As the partial results are released before the actual commit of a long-running transaction, the mechanism for releasing them is called Conditional Commit.

For conditional commit again we use a dependency graph in combination with the wormhole theorem. It is important to note that:

- in the first place two long-running transactions had full invisibility towards each other, therefore the released data-item from the first long-transaction has to be read by the second transaction

- as all the data-items are in the deployment level (recall discussion in Section II, A) they will be created by the transaction in the first place, that means the first operation on a data-item for any long-running transaction will be a write (in fact, it can take place in one of its sub-transactions but this is the primary assumption of SOA).

Therefore, in any conditional commit between transaction $\mathrm{T} 1$ and $\mathrm{T} 2$ there is a write $\rightarrow$ read dependency and as the first transaction is not fully committed, any write operation can create a wormhole $(\mathrm{T} 1<<<\mathrm{T} 2<<<\mathrm{T} 1$ in term of write $\rightarrow$ read $\rightarrow$ write). That is why after releasing the partial result the dataitem will be read-only and this cannot change until the first transaction commits. Note also that the second transaction cannot commit before the first one does and as a result it will have a commit dependency.

For addressing this limitation, we define a C-Lock for the conditional commit of partial results and the dependency graph for releasing these data-items is called External 
Dependency Graph (EDG). In addition to capturing the dependencies on particular data-items released between transactions, this graph also captures the commit dependency. By using the EDG the second transaction can not commit unless it receives a confirmation from the first transaction that it has committed. Figure 12 shows an example of using conditional commit.

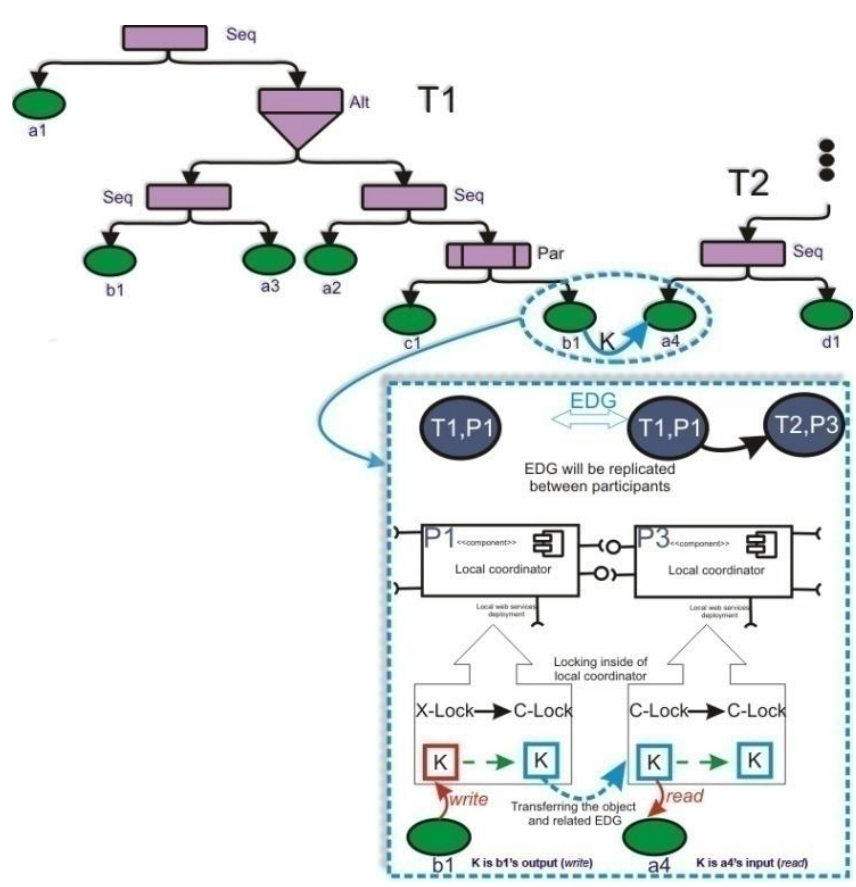

Fig. 12 EDG and C-Lock

In Figure 12 transaction $\mathrm{T} 1$ releases the partial result (dataitem $\mathrm{K}$ ) to transaction $\mathrm{T} 2$. The last participant of $\mathrm{T} 1$ to operate on data-item $\mathrm{K}$ is participant $\mathrm{P} 1$, and this operation is a write operation as explained before. Since $\mathrm{K}$ is to be released to another transaction $\mathrm{T} 2$, rather than inside $\mathrm{T} 1$ which is the case we saw in the previous section, P1 applies a C-Lock to dataitem $\mathrm{K}$ before it is released to transaction $\mathrm{T} 2$ to be read by participant P3. This is recorded in the related External Dependency Graph (EDG) will be created by coordination of Participant P1 of T1 (the participant which has done the last action on data-item K), and the graph is shared by P3 of T2 ( the participant which is going to do the first read operation on data-item K).

We note that in the corresponding $\mathrm{xml}$ schema for the EDG, which can be found following [34], this is reflected by a structure of three elements in the ExternalDepednecyGraph element: Originator (the creator of the data-item in question), Identity (to identify the particular data-item), and ExternalDependency (to identify dependent transactions).

The important point is that the C-Lock has been used for data-item $\mathrm{K}$ and the lock will be inherited by any participants which are going to use the data-item in the sequel (in transaction $\mathrm{T} 2$ or any other that uses it subsequently). In this way, the data-item will remain 'Locked' until the transaction, the one which has created the data-item (called the originator transaction; in our example this is T1), commits. Meanwhile, in terms of abortion in the originator transaction, all dependent transactions should be rollback. In the next section (subsection $\mathrm{B}$ and $\mathrm{C}$ ) we describe the recovery procedure that needs to take place.

\section{NETWORK FAILURES AND RECOVERABILITY}

So far we have been concerned with correctness of our lock scheme used in long-running transactions. Now we turn our attention to failures that may occur at both the transactional and the network levels, and show how these can be addressed in recovering the system to a consistent state.

\section{A. Transactions and connectivity}

We have seen in Section III, B, that the network supporting a digital ecosystem can be conceptualised as the result of several business transactions where each transaction creates a private network, the so-called VPTN.

Conceptually, we consider $D_{U}$ as all Digital Ecosystems, where $T_{U}$ is all of the possible transactions in these Ecosystems and $P_{U}$ all possible participants of the Ecosystem. Each transaction is the result of compositions of services from several participants. This can be described by the pair $\left(t, P_{t}\right)$, where $P_{t}$ is the set of participants which are involved in a transaction $t$.

The universe of digital ecosystems comprises all of the possible transactions and their participants and can be defined as:

$$
D_{U}=\left\{\left(t, P_{t}\right) \mid t \in T_{U} \wedge P_{t} \subset P_{U}\right\}
$$

We define a Digital Ecosystem $D E$, as a subset of $D_{U}$, where all of its participants, by engaging in its transactions, are connected.

Each VPTN can be recognised by the transaction of its participants

where

$$
\left(t, P_{t}\right)
$$

$$
P_{t}=\{\text { Participants involved in Transaction } t\}
$$

In this paradigm, the maximum number of links which a participant may have is given by:

$$
\left|P_{t}\right|-1
$$

In our transaction model, coordination of the underlying services is distributed and addresses both the order and the data dependencies, and hence the actual number of links is always less than this. It is given by:

$$
1 \leq \text { No of links for a node in }\left(t, P_{t}\right) \leq\left|P_{t}\right|-1
$$$$
\text { EQ. } 4
$$

But based on the definition of a digital ecosystem, VPTNs can have overlaps which make a connected network. Therefore, nodes can be involved in several VPTNs and as a result, they will have additional links through participants of different transactions. Thus, a participant $R \in P_{t}{ }_{i}, i=m . . n$, 
which is involved in transactions:

will have links between:

$$
\left\{t_{m}, \cdots, t_{n}\right\}
$$

$$
\begin{gathered}
m+n \leq \text { No of links of } R \leq \sum_{i=m}^{n}\left|P_{t_{i}}\right|-(m+n) \\
\text { EQ. } 5
\end{gathered}
$$

If $R$ participates in different transactions is through different participants. Studies show most business networks follow the power-low distribution degree [16], which means that a very small number of nodes are involved in the majority of the transactions (EQ 5), and even in each transaction they will have the maximum numbers of links (EQ. 4).

Figure 13 shows a simple digital ecosystem, where 'Participant 4' and 'Participant 3', are involved in all of the VPTNs, and in each VPTN they get the majority of links.

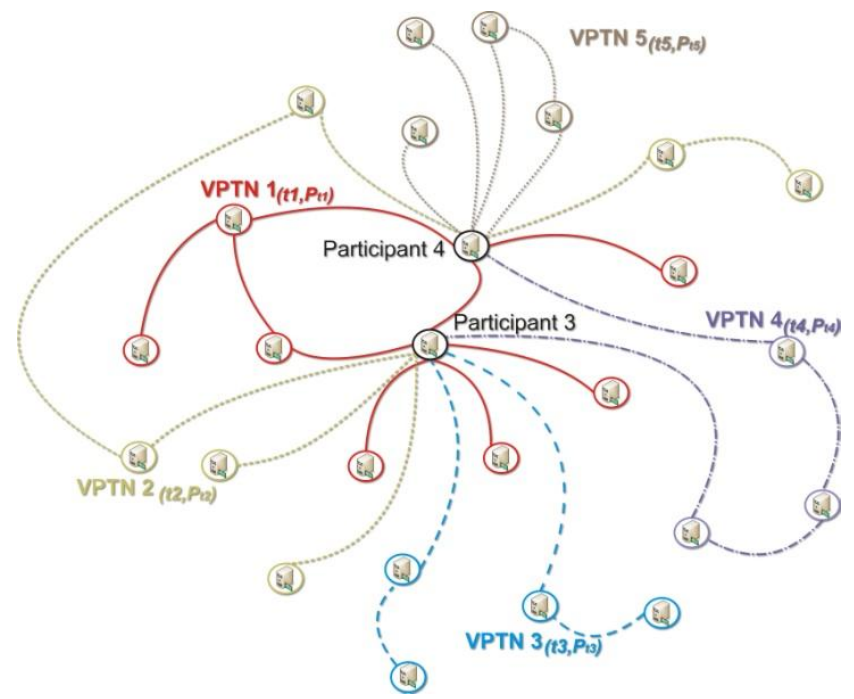

Fig. 13 Digital Ecosystem of connected VPTNs

This has as a drawback any problem in either 'Participant 4 ' or 'Participant 3' (or both) can cause serious disruptions in all VPTNs. Meanwhile a simple failure on 'Participant 4' or 'Participant 3' can fragment the network, which means even if the involvement of 'Participant 4' or 'Participant 3' was restricted to alternative service composition, still the transactions may not be executed. Moreover, since 'Participant 4' and 'Participant 3' have gotten this important role based solely on their business transactions they may not be the best candidate for providing connectivity for the network. In other words, their emergence as a highly connected node has been driven by the volume of transactions they take part in and no other factor. This raises the question of whether it is desirable for a digital business ecosystem to rely on very few nodes in general.

Before addressing such aspects, we show how each VPTN will react to a failure and how it can be recovered, how the cost of failure can be reduced, and how full abortion of the transaction can be avoided. Then, in section D we examine how the possibility for failures can be reduced all together.

\section{B. Recovery Procedure}

We start with the well-known 'Rollback theorem' and build our recovery procedure around the concepts of degenerating the transactions and, of course, avoiding wormholes.

Rollback Theorem: A transaction that unlocks an exclusive lock and then does a 'Rollback' is not well-formed and, is potential wormhole, unless the transaction is degenerate.

As the theorem is well-known, we refer the interested reader to [23] for the actual proof. The important point of the theorem is that we have to degenerate the transaction to effect rollback. For this purpose we can use the logs provided by the dependency graphs described in Section III and trace them. The only caveat is that the digital ecosystem network (of VPTNs) is distributed and therefore there is no centralised synchronisation. This entails that there is a risk for wormholes.

\section{1) Two phase recovery}

For avoiding wormholes, we have designed the recovery procedure in line with our consistency model (logs/locks) for concurrency control. Overall, Recovery Management in combination with the concurrency control procedure runs in two phases:

1. Preparation phase: consists of sending a message (abort/restart) to the participants of all subtransactions that puts them (and their data) into an isolated mode (preparing for recovery). This helps avoid any propagation of inconsistent data and possibility for creating wormhole during the actual rollback.

2. Atomic Recovery Transaction routine: the recovery routine will be run as an atomic procedure that can rollback and cancel deployed services of subtransactions by using correct data-items.

Both phases in recovery management rely strongly on tracing the corresponding dependency graphs. This is where the necessary information for finding the changes on dataitems, in different participants, and undo them and bring back the system to a consistent state. Figure 14 shows a sample scenario that extends that presented earlier in Figure 11 and Figure 12. 


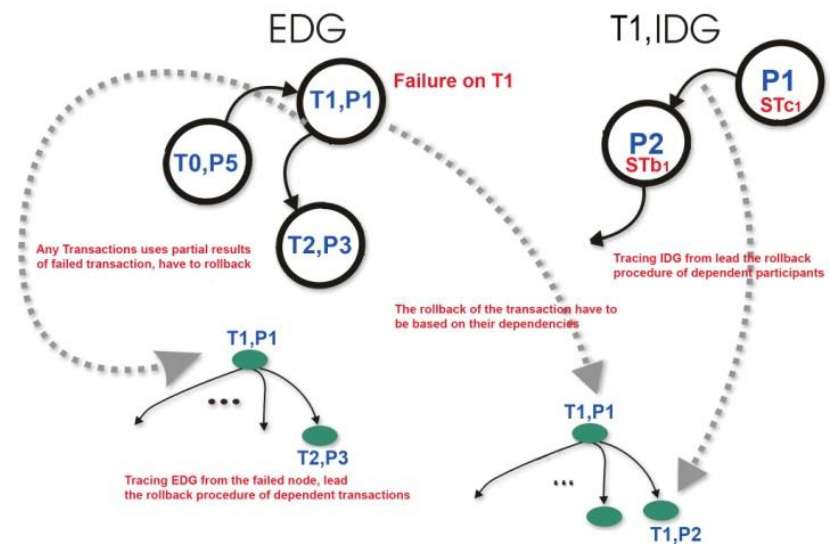

Fig. 14 Tracing dependency graphs for Recovery

According to Figure 14 a failure happens for $T 1$ while participant $P l$ was trying to execute the transaction. The participant $\mathrm{P} 1$ has to stop any further progress on $\mathrm{T} 1$ and uses its EDG and IDG for informing about the failure on T1. As shown in the figure, participant $\mathrm{P} 3$ uses some results from P1 for transaction $\mathrm{T} 2$, which means $\mathrm{P} 3$ has to start a similar procedure for transaction T2. This is the external dependency. Participant P3 now uses its IDG, which indicates that the participant $\mathrm{P} 2$ needs to be informed for stopping the potential execution of transaction $\mathrm{T} 1$ (in this case, it will the subtransaction STb1 of transaction T1). In fact, it has to inform any dependent transaction or sub-transactions by checking its related graphs to cater for all internal dependencies. Now for stopping the transaction progress (isolation of $\mathrm{T} 1$ affection) upon failure, we need an internal structure inside of the local coordinator.

\section{2) Isolated Recovery}

We have seen that the first phase of Recovery Management tries to just isolate the damaged (or failed) part of the system by distributing a message that can isolate all worked dataitems of transactions of sub-transactions. We have also seen that in the transaction model, the I-Lock and C-Lock are locks which release data-items which can be the most problematic part of transactions in recovery. Tracing the relevant IDG and EDG can reveal these vulnerable data-items.

We introduce R-Lock as a fully isolated lock, which can be used just for rollback purposes. As part of tracing and stopping the progress of a failed transaction (and any affected transaction by the failed results of the transaction, in terms of partial results) we convert the data-items locks to R-Lock. Figure 15 shows the complementary routines, which in combination with Figure 14 can show the first phase of the recovery.

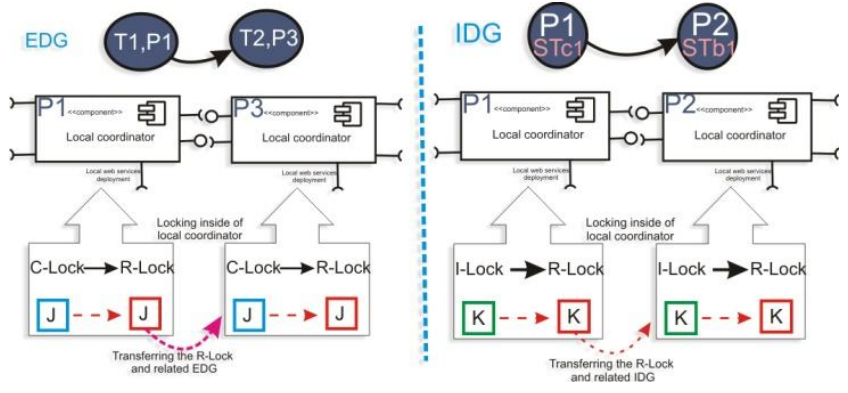

Fig. 15 R-Lock and Isolated Recovery

After using R-Lock (Fig. 15) the second phase of the recovery routine can be seen in the context of conventional shadow-based recovery. As the local agent of each participant keeps transaction information, such as changes and updates (even the committed transactions will have been archived) in its local repository (Section III and Fig. 5), the previous content of data-items can be retrieved and the deployed services can be cancelled. It is important to notice that for doing this the participant does not need any external help of other participants or synchroniser, and theoretically the second phase can follow the first phase without waiting for confirmation from all other participants.

However, a practical consideration has to do with what will happen if the failed transaction could not reach some of relevant participants. More generally, how can the possibility for full recovery be reduced, since it can be quite costly for the digital ecosystem. We attempt to address the second concern first.

\section{Forward Recovery, for reducing the recovery cost}

Full recovery can be costly in terms of resources, delays, business relations and so on. Further, we have seen that in a digital ecosystem dependencies may also exist across transactions so the effect of a recovered transaction may be magnified. For this reason it is desirable to avoid full recovery wherever possible. One way to do this is to design transactions with a number of alternative scenarios of execution. For doing so, we introduce 'Forward Recovery' which is a mechanism for avoiding full recovery. The aim is during recovery failure to explore whether there is any possibility for successfully terminating the transaction following a different execution path to the one originally deployed, instead of rollback of the whole execution tree.

Forward recovery is can be an option when there are alternative service compositions in the transaction (recall Section II, A-1). By failing one sub-transaction of an alternative coordinator, that specific sub-transaction should be fully rolled back (but until the point of an alternative service composition) and then the alternative scenario can be tried by the participant's coordinator to commit the transaction with its other sub-transaction(s). 


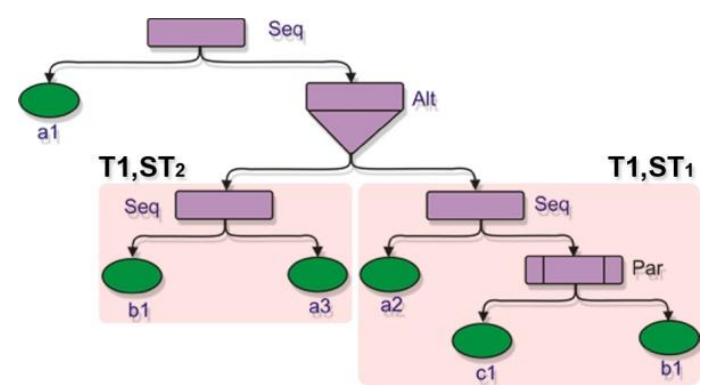

Fig. 16 Alternative Composition and Forward Recovery

Figure 16 shows an example in which transaction $T 1$ is using an alternative service composition $(A l t)$, where there are two alternative paths; one following sub-transaction $S T 1$ and one following $S T 2$. So far, we used the bottom part of $S T 1$ in Figure 11 and 12 to illustrate the release of a data-item inside of the transaction, and in Fig 14 and 15 for demonstrating the recovery procedure. If we assume $S T l$ is attempted to execute, if a failure happens (for example in Fig 14 the relevant participant could not fulfil the execution of $S T c 1$ ), still we have the alternative path which is following sub-transaction ST2 (in the left of the alternative service composition typeAlt).

For doing so, $S T 1$ must be rollback, as was shown in the Fig 14 and 15, by using IDG and EDG, the internal and partial result should be traced and rolled back. After this, instead of continuing the recovery procedure and aborting the Transaction T1 completely, the second path of alternative service composition can be executed (ST2).

\section{Network failures and transactions}

As the digital ecosystem is a distributed environment, where each participant can work in a loosely-couple manner, the probability for failure in the network is inevitable. These failures are directly related to network connectivity - when the network connectivity is low, the probability for disconnection will be increased. We provide two mechanisms in our approach for addressing this challenging problem:

- Reducing the probability for network failure

- Providing the automatic procedure, in cases of unexpected network failures

In the beginning of this section we have shown a simple comparison for the best and worst case of connectivity for each participant. For reducing the probability for network failure, we try to provide a growth model to increase the connectivity. This is discussed in further detail in Section V and VI. Here, we introduce a mechanism for unexpected network failures.

When a participant involved in a transaction does not receive an expected response from another participant, it should be able to make decision despite that response. As the first assumption, each participant, in terms of the transaction context, considers an 'Expected Response Time'. In each step of the long-running transaction execution, if a participant has not received any response during this time period, it automatically freezes the data-items related to the transaction. In this way, until clarifying the status of the transaction the unnecessary execution of it will be suspended, the network resources will be saved and if there is failure, inconsistent data-items will not be spread in VPTN and possibly the whole ecosystem.

For this temporary freezing of the data-items we introduce T-Lock ('Time-out Lock'). The T-Lock is rather like giving a time-out before rollback of a data item. The access to the data item will be limited until a deadline (time-out). If during this deadline the other participant responds, the original lock will be restored, i.e. T-Lock will be converted to the original lock. Otherwise, after the time-out elapses the recovery procedure will be started. The 'Expected Response Time' for participants also depends on the network parameters and can be fixed statically based on the digital ecosystem's network characteristics. The 'Time-out' of the T-Lock is related to the transaction expected life time and it can be varied according to the transaction context.

\section{Stable Digital Ecosystem Network Structure}

The purpose of a business network is to enable networked organisations to engage in distributed business transactions [20], [14], [4] that realise their core business activities. As we have seen in the previous sections in terms of business transactions this means stronger interconnectivities for VPTNs. This is achieved when a transaction's participants can avoid failure at the supporting network level and/or alternative paths being reachable whenever service unavailability is experienced or failure in one of the participants in alternative scenarios. This may lead us to increasing the local connectivity in each transaction, but the effect and side effect of changes will direct us to take into account a measurement for stability and apply any increases in terms of this measurement. This provides a dynamic and extensible method for creating a stable Digital Ecosystem that emerges through the long-running transactions that correspond to business activities between participating organisations.

\section{A. VPTNs interconnectivity and network connectivity}

Increasing the connectivity between participants of a transaction prevents certain types of failures in the transaction, predominantly those which are the result of the network disconnections. At the same time, alternative scenarios in terms of alternative service compositions (Section II, A- SAt and PAt) rely on available connectivity between alternative paths (between different participants) in the network. This means increasing VPTN interconnectivity helps to provide a better chance for forward recovery (recall discussion in Section IV, C), and as a result avoid a full recovery even when some participants failed to provide their services. Before 
describing how VPTN interconnectivity is dealt with in our framework, we present the general mechanism for link replication in the local software agent of each participant.

\section{1) Link replication, and connectivity}

Normally the connections (links) to other participants of the digital ecosystem have been established by the 'global service repository', where the address of other service providers (participants) and the description of their services have been kept. For inserting (or modifying) a new participant to the repository and its services, the 'web service information investor' component will be involved. For introducing the participant to another participant the 'web service promoter' will be used.

It can be seen that for increasing the connectivity we use three components of the component-based design of each participant. Figure 17 shows the relationships between these components of three participants (their software agents). Participant B ('Agent B'), receives all of the connections of Participant A ('Agent A') through its 'web service information investor' when participant A ('Agent $A$ ') provides them through its 'web service promoter'. Similarly, participant B ('Agent B') provides its connections (links to the other participants) to the 'web service information investor' of participant C ('Agent $C$ '). We call this procedure Link Replication. It is important to mention that it is possible to have partial link replication where there is no need to replicate or pass to other participants all of the connections of a given participant.

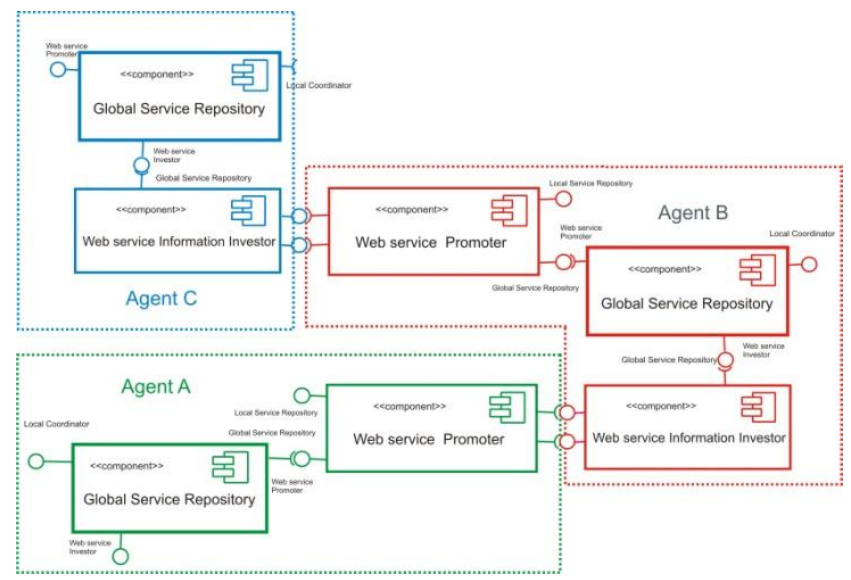

Fig. 17 Link Replication

\section{2) Fully connected VPTN and Digital Ecosystem}

As we mentioned in Section VI (D) and the beginning of this section, one of the significant risks for the VPTNs of a digital ecosystem (transactions of the network) is disconnection between participants of a transaction which amounts to low connectivity inside the VPTNs. It seems the primary solution for the problem is to use the link replication procedure between the participants. By repeating link replication in each participant, within a limited time, all participants in a VPTN will be connected together. As a result we will have a fully connected VPTN, which if it is built based on transaction $t$ and participant $P_{t}$ each node will have $\left|P_{t}\right|-1$ links.

Where this seems like an ideal solution for each VPTN the result can be devastating for the digital ecosystem and consequently the majority of transactions could be failed. As a digital ecosystem is a connected network through its transactions (Section II, B), the VPTNs have overlaps on some of the their participants (there are some intersections between different VPTNs' participants) and as mentioned in Section IV, A, studies show most business networks follow the powerlow distribution degree [16], which means:

a) The digital ecosystem relies on very few participants (nodes) to stay connected

b) And these small numbers of participants are involved in the majority of the transactions, i.e. these few participants will be in most VPTNs.

Now if we apply link replication for each VPTN $\operatorname{VPTN}_{i}\left(t_{i}, P_{t_{i}}\right)$ where $\operatorname{VPTN}_{i}$ is VPTN of transaction $t_{i}$ and its participants are $P_{t_{i}}$ ), each participant in the VPTN will have

$$
\left|P_{t_{i}}\right|-1
$$

Therefore a participant $R$ which is involved in transactions

Will have up to

$$
\left\{t_{m}, \cdots, t_{n}\right\}
$$

$$
\sum_{i=m}^{n}\left|P_{t_{i}}\right|-(m+n)
$$

links.

Based on the second point above (point ' $\mathbf{b}$ '), very few participants are involved in the majority of transactions. Therefore by applying link replication in this way this small number of participants will have a very large increase of links. This increases their traffic dramatically and it is highly probable they collapse as a result, which means a potentially large number of transactions will be failed. More importantly, based on the first point above (point 'a'), as the digital ecosystem relies on them to stay connected, the whole digital ecosystem will be fragmented. Figure 18 shows this situation which his generally rather difficult for a network to recover from.
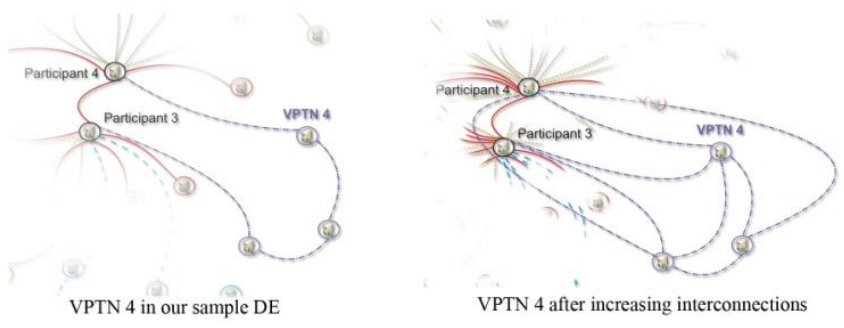

Fig. 18 Fully disconnected VPTNs

On the left side of Fig 18 VPTN4 from the sample digital ecosystem presented in Figure 2 and 13, is shown, and on the right-side we can see the result of link replication on all 
participants of the VPTN4. As link replication in a similar way has been applied for all other VPTNs, participant 3 and participant 4 which are involved in several transactions face the large increases of links which can bring traffic complexity. While the link replication itself seems quite useful for increasing connectivity, the way and on which participant it is applied can be crucial for the general performance of the digital ecosystem (and even each VPTN).

Before investigating some measurements for a more careful application of link replication we try to review the other conventional approaches in network interactions.

\section{3) Conventional peer-to-peer solutions}

The oldest solution to the problem of connectivity is to supply a powerful central coordinator that manages the whole network and keeps all information about all participants. However, this solution has the classic problem of a single point of failure as well as high cost for providing and maintaining the centralised unit. Note the cost increases as the number of nodes and associated network traffic increases.

A popular solution to the connectivity problem which is used in several P2P networks (e.g. [5], [6]) consists of introducing an extra layer to the network, the so-called super peers. These are essentially decentralised servers, each managing a significant part of the network (number of participants), and have strong links to each other. The primary necessity for having super peers is providing stable nodes which are online all of the time. This means super peers are expensive nodes with costly maintenance requirements. Furthermore, during peak time the pressure of high traffic can result in a bottleneck on super peer nodes. At off-peak times the powerful super peers will still need to be online and monitor the whole network, thus processing redundant data and producing overheads waste.

It should also be noted that the resources are used for facilitating network operation management tasks. When considering such a solution for a digital ecosystem environment involving SMEs, the question arises as to who is going to provide such nodes? Even if it were possible to find suitable SMEs willing to provide permanent nodes as super peers, these may change their business model and after some time may not find it useful to provide a permanent (and expensive) node anymore.

Perhaps even more importantly, the super peers solution results in a static topology for the network as these nodes are pre-selected and their role is pre-determined in the network. This is by no means satisfactory in a highly dynamic environment of a digital ecosystem where the idea is that the network topology changes continuously to adapt to its very usage and demands of the participating entities. The evolving nature of the $\mathrm{DE}$ is intended to reflect the congestion of network packages and nodes that change from time to time.

On the other hand, models which provide self-management capabilities at the service level [7], [8], [9], [10] and Quality of Service (QoS) at the virtualisation levels [11] can be seen to be another extreme solution for Digital Ecosystem environments. But the network resistance against failure on the collaborative business activities for long-lived transactions from failures, (in the face of the highly dynamic business models of SMEs) has not been solved, which cannot be expected to provide the necessary permanent platforms for a connected network.

\section{B. Towards a connected Digital Ecosystem}

In this part, we try to provide a constructive solution for using the link replication mechanism to improve connectivity inside VPTNs and present the first step towards a stable digital ecosystem. In general, we can say the best candidates for link replication inside each VPTN and connecting VPTNs together are the most stable participants (nodes) in each VPTN. Connecting these participants and the link replication can be done by using the 'Global Service Repository' of each candidate-participant from each VPTN (it has been shown in Fig. 17, Section V, A, 1).

However, we cannot warranty full stability of the network and still cannot avoid the occasional fragmentations. Even in the best case, this is still is dependent on each candidateparticipant's availability and if the total online time of all stable nodes cannot cover the full 'active-time' of the network, the network will collapse for some period of time, precisely that in which all candidates are not available. Therefore, first we try to introduce a measurement for node stability, and then use this measurement in finding the stable participants in each VPTN to cover the network's 'active-time'. Active time we refer to the time period when any none-zero number of participants are active and working - if the digital ecosystem is large, practically this has been considered 24 hours [14], [2].

\section{1) Stability measurement for nodes}

Since we are dealing with connectivity as a means of avoiding disconnections and fragmentation of the network, we need a measurement for node stability. It would be unreasonable (and not feasible) to expect participants (nodes) to be online all the time and thus stability is determined on the basis of declared availability.

For finding a more precise and computable measurement for node stability, we introduce the so-called Expected Availability Time (EAT). This is the time the node is expected to be available and online in the network. Figure 19 shows an example of EAT for a node in the network. 

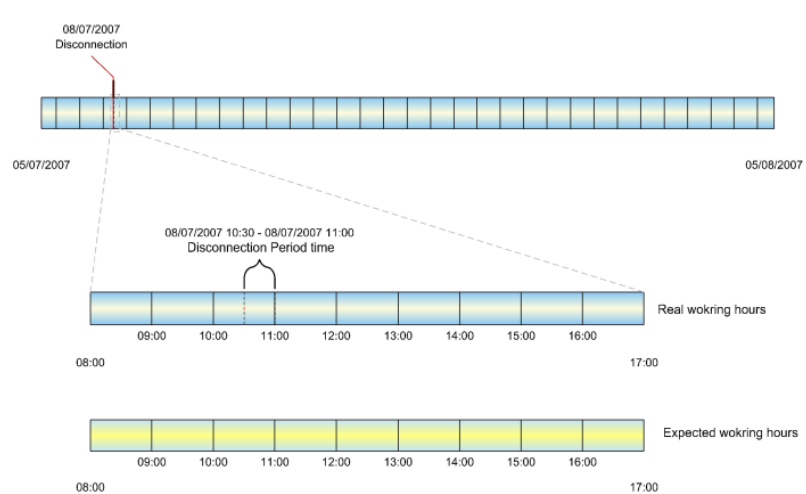

Fig. 19 Expected Availability Time

The node stability is then calculated as the actual availability of the node against this expected time. These are typically different, since during its EAT the node may experience disconnections.

This will reduce stability (reliability) of the corresponding node in the final selection. This notion of stability can be simply calculated as below:

$$
\text { NodeStability }=\frac{\text { EAT }- \text { DisconnetionPeriods }}{E A T}
$$

It can be seen that NodeStability $\leq 1$ and the closer NodeStability gets to 1 for a node, the more stable the node is (which can be understood as more reliable or predictable). As an additional parameter we have also considered a traffic limitation on the participants. If the infrastructural traffic reaches a specific percentage (' $K$ ') of a platform bandwidth, and this can be varied depending on the ecosystem's business cluster, the participant is regarded as disconnected and this will increase its 'Disconnection Periods'. This can be justified since the priority for each participant is its transactions and we do not want to overload the participants by infrastructural traffic and cause potential collapse and transaction failure.

In any case when a participant is involved in some transactions it is still indirectly helping the infrastructure by its VPTN links. This is one aspect that distinguishes our model from other approaches that may use a mechanism similar to link replication but without considering the participant's situation, which has been discussed at subsection A-2, of this section.

In our latest simulation which his described in greater detail in Section VI, A, the participant's bandwidth has been treated as a random number between $500 \mathrm{~kb}$ to $4 \mathrm{mb}$ and the maximum percentage of the infrastructural traffic is $\% 30$ of the platform bandwidth but this can vary depending on the environment and average transactional traffic of VPTNs.

For calculating the stability function of a participant in the first instance we use VPTN neighbourhood voting algorithm (where each participants in a transaction calculates its other nodes of the VPTN during each transaction life-time) to finalise this. The average availability in overlapping transactions can determine the actual availability.

We consider alternative methods for one of our implementation plans in Section VI, B, which relies on automatic calculation of availability inside of the coordinator of a transaction and this will be calculated based on overloading traffic more than ' $\mathrm{K}$ ' percentage of the participant bandwidth or disconnections from Internet.

At the moment we have considered EAT as a part of SMEs business model which is given by each SME on joining the network. Hence, this is fixed or can only change on the account of the SME providing it. It should be noted that other approaches can be considered for calculating the EAT - for example, it is possible to use an algorithm based on their VPTNs' actual life time (their transaction life-cycle) or network neighbourhoods' estimations for calculating EAT which would allow it to vary over time.

\section{2) Permanent Clusters and Virtual Super Peers}

As mentioned before, in contrast with conventional super peers, we try in our network design to move towards a more dynamic architecture which does not rely on just a few permanent nodes. Central to our approach is finding permanent clusters on the network. More specifically, we are identifying aggregations of stable nodes, where node stability is determined as in the previous section. For doing so, the most stable nodes from different time zones must be chosen, in a way that they cover the digital ecosystem's 'active-time' (for reasonably large ecosystem 24 hours). More specifically, we are trying to find permanent clusters through the most stable nodes.

The important part in determining permanent clusters is discovering different aggregations of these time zones which can cover 24 hour availability. Any union of the stable nodes in the aggregations (which provides the full 'active-time' - 24 hour availability coverage) are actual permanent clusters. Figure 20, shows the simple situation in which the most stable nodes have been selected from two sets of time zones which can cover 24 hour service availability to form permanent clusters.

\section{Virtual Super Peers}

By using stable nodes from permanent clusters, as shown in Figure 20, we can create Virtual Super Peers (VSPs) which are effectively permanent clusters of nodes in the network. These can provide the desired stability for the digital ecosystem. The strong connection between the virtual super peers themselves on one hand and the connection between them and their nodes decrease the probability for fragmentation. Depending on the level of reliability required for the network, it is possible to include further redundant stable platforms from each available time zone. For example, in Figure 20 we have included two stable nodes from one time-zone and three stable nodes from the other one (the green and creamy coloured signs show different time zones). 


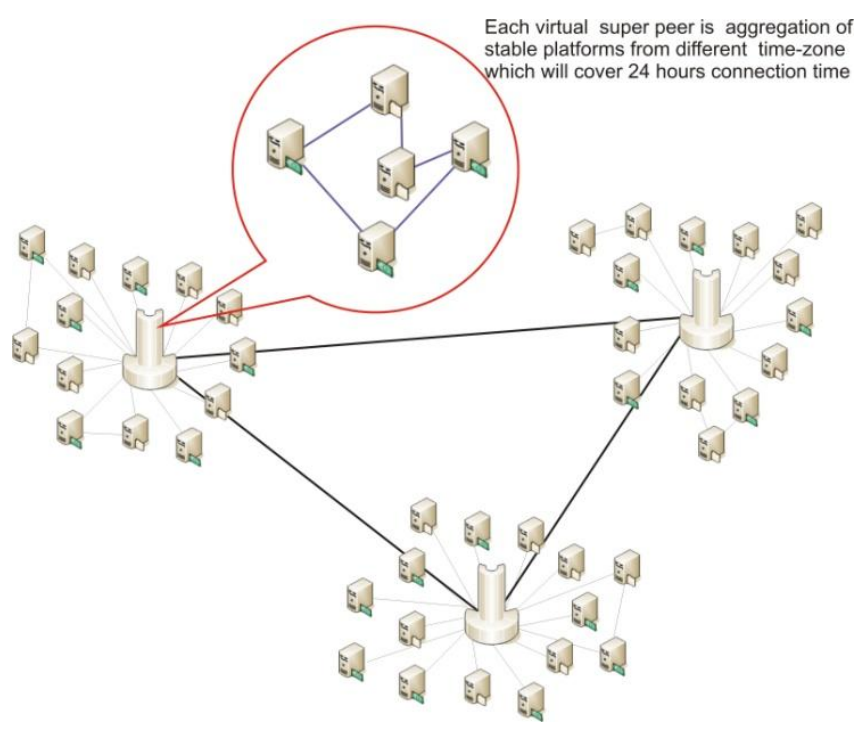

Fig. 20 Permanent Clusters and Virtual Super Peers

In this manner, the good connectivity can cause more reliable transactions at the VPTNs level. Meanwhile the traffic is spread over the virtual super peers and there is less risk of bottleneck at peak time. Participants (nodes) within a virtual super peer need to keep information only about nodes in their cluster and about neighbouring VSPs so at off-peak time the amount of redundant information processing is reduced dramatically as compared to the classical Super Peers solution.

Since choosing stable participants (nodes) is done based on the stability measurement which is given by a function of EAT and the Disconnection Period of a node during EAT, whose value varies over time, is a dynamic process and hence it the virtual super peers are also formed dynamically. This means the topology can change from time to time and new nodes can be added to the permanent clusters as the structure of the virtual super peers changes. A node can become part of a virtual super peer, when its node stability increases and overcomes some threshold, and nodes that are super peers may not be able to cope with the increased number of connections they get, and possibly increased number of transactions they perform and lose their virtual peer status. Within a digital ecosystem for business, SMEs would be expected to invest at that time (in hardware, processing power, bandwidth etc.) and become again part of a virtual super peer in future. It is in this sense that the topology evolves to reflect the usage and demands of the participants who benefit from and contribute to the 'sustainability' of the network.

Additionally, network congestion can change the maximum level of node stability (recall the discussion in sub-section A) which in turn affects the selection of the most stable nodes in forming the permanent clusters. High congestion of packages can increase or decrease network reliability (higher traffic on few virtual super peers can potentially create a bottleneck and even cause fragmentation). In a digital business ecosystem, the best part of the traffic is the result of business activities which are effectively long-lived transactions. These have been virtualised in VPTNs and therefore, using the effect of VPTNs for making VSPs and their client nodes, can increase stability of each virtual super peer.

Furthermore, we expect a reasonable cluster coefficient on the account of having VPTN as the main building block which we have seen is formed from a transaction. This means its participants are in relevant domains - by connecting them to several VSPs we actually increase the probability for that. We also expect a fair distribution degree on the account of propagating links to VSPs. This means that instead of being concerned with individual links for each node, aggregate links of VSPs come into play.

Finally, reusing business activity results (or service-on-fly as result of composite services [32]) and explorative service composition [31] are other factors which can be considered for higher performance within a digital business ecosystem and can provide potential for creating so-called virtual vendors [14].

\section{The dynamic mechanism for choosing VSPs}

In the first step, the most stable participant in each VPTN (participants of a transaction) should be selected for keeping vital information about the transaction and its participants. In this sense, the network provide a level of durability with minimum cost from participants and it provides a greater chance for forward recovery even in terms of failure in one of participants of a transaction. Effectively, this makes our mechanism described in Section IV, subsection C, fully effective with regard to what is referred to as (in purely transactional literature, the solved problem is called) omitted results, which is a problem relating to preserving as much progress-to-date as possible in the event of aborting a transaction (the details about complexity of the problem can be found in our previous work [35]).

In the next step, by connecting the most stable nodes of each VPTN together, the first level of strong connectivity and suitable nodes for VSPs are created. Figure 21 shows the internal structure of each VPTN and the connection between VPTNs. The internal structure of VPTN contains a lot of information from the transaction level such as log structures, lock schemes for ensuring consistency in recovery mentioned above, local coordinator design, formal analysis of the required interactions and recoverability [19], along with alternative scenarios for forward recovery. Now by using the most stable node, we let the optimisation in transactions to be performed and any waste of resources as the result of weak connectivity will be avoided. 


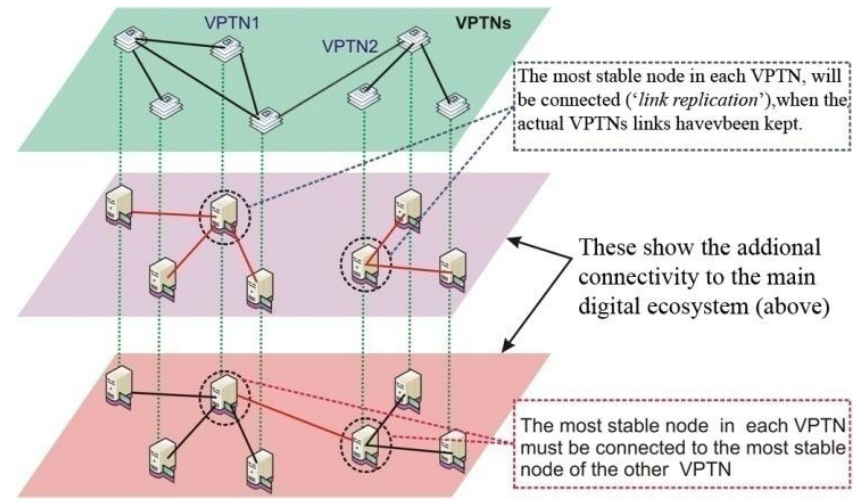

Fig. 21 Dynamic Mechanism for increasing stability

The direct effect of connecting VPTNs together is raising the cluster coefficient of the network. Conversely, connecting the most stable nodes of VPTNs together provides the opportunity of choosing the best candidate locally between these stable nodes for the permanent cluster. Choosing nodes of the permanent cluster in this way results in a virtual super peer that provides fair traffic distribution at the VSN level (each virtual super peer will take care of its local VPTNs). The main concept behind forming permanent clusters stays the same, i.e., selecting the most stable nodes from different time zones which can cover 24 hours online time.

\section{E. The model in practice}

As the most stable node in each VPTN is the best candidate for keeping the transaction information, the corresponding business activities will have increased levels of reliability. The fact VPTNs are used initially in the design of the business network, and are connected through their most stable nodes which are determined dynamically, allows in most cases the candidate platform to avoid the full rollback or compensation of the transaction when some participants of the long-lived transaction get disconnected mid-way through its execution. This has been considered in the design of the recovery mechanism for the digital ecosystem (Section IV).

Another expectation of the proposed network infrastructure is reducing the possibility of fragmentation. We have seen that we are dealing with a highly dynamic transactional environment where there is no central point of control. The current model can fulfil the requirement at the theoretical level. It would however be desirable to be able to somehow guide the way this topology evolves. In the next section, we try to show a realistic simulation which can compare the theoretical behaviour and practical status of the network in different situations. Furthermore the current roadmaps and prototype implementations are described.

\section{IMPLEMENTATION EXPERIENCES AND ROADMAPS}

The first implementation of a Digital Business Ecosystem was relying on FADA nodes [14] as the core infrastructure for the network. Items (service proxies) are registered in any node of the FADA cloud, and they can be searched for starting from any node in the FADA cloud. The FADA nodes create a free graph, i.e., the topology is not enforced and not even known. As a result FADA nodes are relying on node-interactions (transactions) for creating links. In this sense, the network design we propose builds on the principles of FADA, but is extended with the design concept of dynamically formed Virtual Super Peers.

As mentioned in Section IV (A) the expected results from such a network is the power-law distribution degree (scalefree network). In what follows we start by examining the FADA network (a network topology which just relies on its transactions and potentially creates a power-law distribution degree) and model it. Then we continue by introducing the current stage and ongoing implementation of our model.

\section{A. Simulation and expectation}

\section{1) Analysing the result}

In the first step, by using the frequency distribution of links per node we try to clarify the distribution of links among nodes. This enables us to compare similar snapshots of nodes for FADA and our network in a similar situation.

For clearer comparison, we use cumulative frequency analysis to examine the network situation during a critical situation. For example, in the next section we investigate the impact in case of failure of crucial nodes on the network, and specifically its connectivity. Despite of the facility for comparison between two different types of networks and questioning the topology reaction in a decisive situation, this can steer us to formulate the confidence statements (as the indication for the reliability of network) as future work.

\section{2) A conventional Digital Ecosystem}

FADA [15] as a conventional implementation of a digital ecosystem is a scale-free network, which is relying on a few hubs. The effect of this for SMEs is an inevitable bottleneck at peak time. As a simple example Fig. 6 shows how the core infrastructure may indeed rely on a few hubs. This not only causes high traffic on peak time (and as a result instability of hubs during this time), but also the possibility of fragmentation and creating islands in the network grows. Especially when we take into account the regular unavailability of SMEs based on their business model and regional working hours. Fig. 22 shows the relationships between the number of nodes (on the vertical axis) and the number of links (horizontal axis). Clearly, a few number of nodes have the most number of links (high degree) while the majority of nodes have just a few links (and these few links mostly end up to a high degree node). 


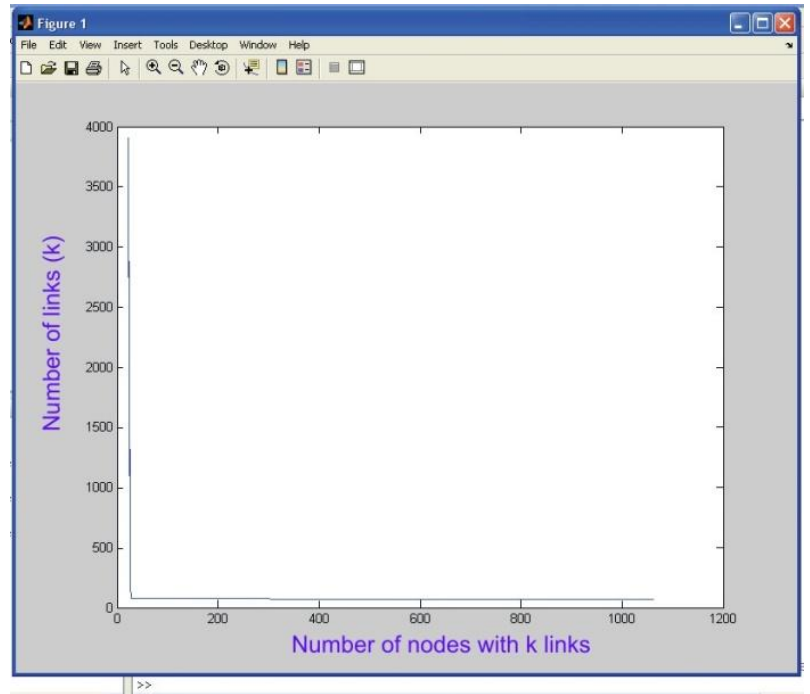

Fig. 22 Distribution degree of a conventional Digital Ecosystem

Based on Fig 22, in a conventional Digital Ecosystem, a few number of nodes have the most links ( 8 nodes have more than 2500 links) and majority of nodes have a few links (more than 4000 nodes with less than 5 links). As a result the network has to rely on a few hubs which may not be the most reliable nodes. Conceptually, they have been hubs because of the high number of business transactions which they participate in - the result is close to Section VI, A-2 estimation.

As a typical scale-free network whose distribution degree follows a power law distribution, any failure (or high traffic complexity) on hubs can cause immediate disruption at the transactional level (abortion of the majority of transactions) and fragmentation of the network. These problems are addressed in our current design and the use of virtual super peers shows significant improvement on the infrastructure of the digital ecosystem. As demonstrated in the next section, the dynamic topology of the network can react in response to failures or attacks on the virtual hubs.

\section{3) Dynamic VSPs model for a digital ecosystem}

By using a dynamic measurement for choosing nodes in VSPs, the dependency on a few nodes with higher distribution degree decreases dramatically. Fig. 23 shows an example of a DE where links are propagated on different nodes.

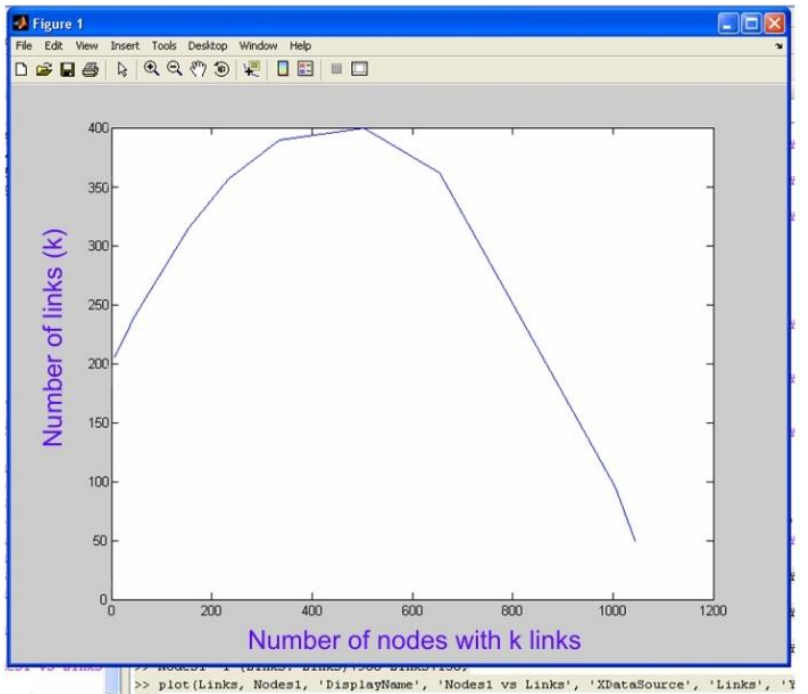

Fig. 23 Distribution degree of digital ecosystem with VSPs

Our primary results with the same number nodes (5000), shows spectacular shift in comparison with the typical FADA infrastructure (compare with Fig. 22) when more than 800 nodes have more than 350 links (nodes which made virtual super peers) and even their neighbouring nodes (which have node stability measurement close to 1 ) have large number of links (about 900 nodes with more than 300 links). These become good candidates for joining the virtual super peers by substituting existing member nodes during failures or attacks on current VSPs. As a result, using Virtual Super Peers and relying on node stability, rather than the business activity, provides a fairer distribution degree. As depicted in Fig. 23 Considerable numbers of nodes have higher number of links.

\section{4) Failures and reactions}

Fig. 24 shows the result of a simulation of 800 simultaneous failures to all VSPs nodes (nodes in permanent clusters forming Virtual Super Peers). These nodes lose all of their links and then only through performing a few business transactions they rejoin the network. As a result of these failures they have very weak stability (NodeStability close to $0)$. Therefore, despite of their transactions they will not receive link replications and still have a low number of links (less than 75 in the simulation of Fig. 24).

Meanwhile their neighbourhood nodes have been substituted in the VSPs structure and their links increased (Fig. 24 shows this effect). But the interesting point is that still the network has not suffered any fragmentation. We may experience some longer response time on business transactions execution but the Digital Ecosystem does not suffer the full failure. Typically, such a severe attack resulting in this type of simultaneous failures would cause several fragmentations. 


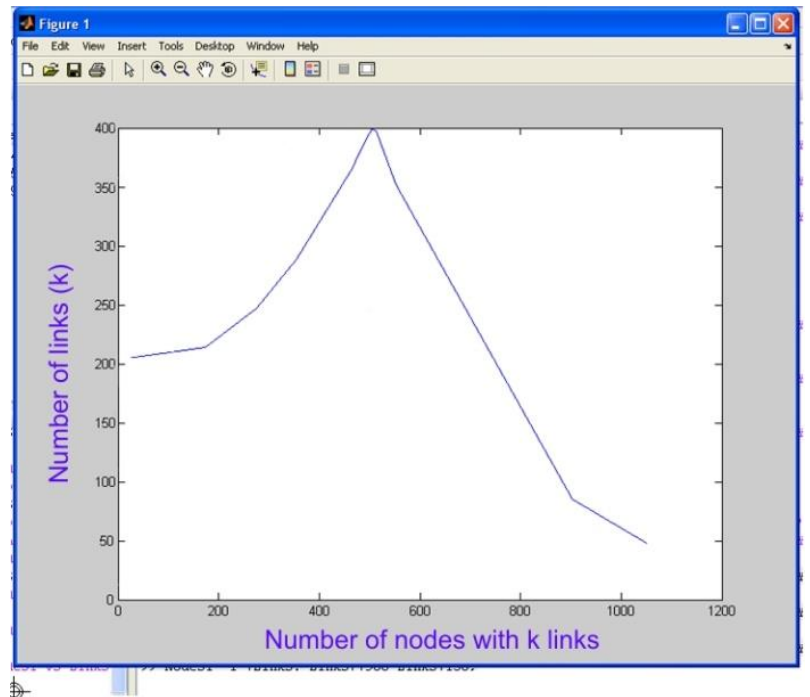

Fig. 24 Distribution degree after failure on VSPs

\section{B. JXTA and DE model}

In this section we give a brief account of our implementation experiences with this work so far. We have seen that our P2P network design is based on the local networks that result from executing long-running transactions involving the deployment of services. The transaction model is mostly concerned with interactions in terms of the coordination of distributed services. The characteristics of the model, outlined here and described in full detail elsewhere [DEST08-trans,FESCA08], are useful in setting up more general distributed and concurrent interactions.

In collaboration with IPTI 1 ('Instituto de Pesquisas em Tecnologia e Inovação', see http://ipti.org.br/) we have been looking at providing an implementation of the transaction and P2P support in a digital environment for business communities.. Our coordinated efforts are directed at implementing aspects of the $\mathrm{P} 2 \mathrm{P}$ network design described in this paper and exploiting the characteristics of the transaction model, mostly in terms of interaction-based service composition and the fine-grained lock scheme, in supporting complex interactions within IPTI's online collaborative platform guigoh (http://www.guigoh.com ) which is used for social networking and e-learning activities. The guigoh platform provides a number of tools such user-created communities, collaborative editing, online conferencing to name a few, but at the moment relies on a centralised P2P network to provide such services. . In the first instance we have been looking at reducing the traffic complexity of the interactions and adding provision for business services. The longer term objective is to migrate guigoh to a purely distributed P2P network that is based on the VSP design solution described in this paper.

The first implementation uses JXTA protocols, which are defined as a set of XML messages which allow any device connected to a network to exchange messages and collaborate independently of the underlying network topology (https://jxta.dev.java.net/). The current available implementation is concerned with the transaction context, through sample service-oriented scenarios, where the main services are optimised for creating parallel, sequential and alternative compositions of virtual online conferences. This has included the full distributed transactional communication (exchange of messages, initiating a transaction, and terminating the transaction). The $\mathrm{P} 2 \mathrm{P}$ relationship between participants and their services has been supported in a purely loosely-coupled manner using the JXTA P2P framework. A prototype of this work, together with preliminary documentation, can be found in the open source project 'flypeer' [38] and can be downloaded from (http://kenai.com/projects/flypeer).

In the next steps, we plan to extend the implementation prototype by considering more complex scenarios and introducing additional traffic complexity through incorporating more heavy services, such as voice, video streams. At the same time, we are looking at introducing a larger number of transaction participants and work is in progress in integrating the user interface for monitoring the model.

\section{The model on an XMPP implementation}

Our work on distributed coordination of long-running transactions involving the deployment of services started in the Digital Business Ecosystem (DBE) project [17]. The support for a distributed transaction model initially targeted the DBE Studio [30] which can be understood as a service container for search and deployment of services from various service providers, and was specifically targeted at SMEs. The industrial strength implementation of the DBE Studio (done by TechIdeas, see http://techideas.es/) used the so-called FADA network [15]. The FADA nodes create a free graph, in other words the topology is not enforced and not even known [http://fada.sourceforge.net/].

Experience with FADA has shown the network, as a conventional scale-free network [Barabasi], is relying on few hubs. This has potential pitfalls as discussed before (Section V B2). Subsequent analyses and further experimentation under the real DBE studio implementation revealed certain problems relating to connectivity and fragmentation, but also high traffic on peak time (and as a result instability of hubs during this time). Such aspects are particularly important, especially for digital ecosystems formed by communities of SMEs and have been highlighted by our simulations reported in Subsection A of this section.

To address such issues the implementation work was steered towards using XMPP (http://xmpp.org/tech/) protocols and a first implementation of the network suing XMPP can be found in the Sironta platform (see http://www.sironta.com/). XMPP at its core is a technology for streaming XML over a network. Our transaction framework is concerned with optimising transactions in terms of the XML-described 
transaction context and the consistency model for the required interactions. Further work is in progress on integrating these characteristics in the next version of the XMPP implementation in order to provide a customised infrastructure that includes support for long-running transactions in the service-oriented platform Sironta.

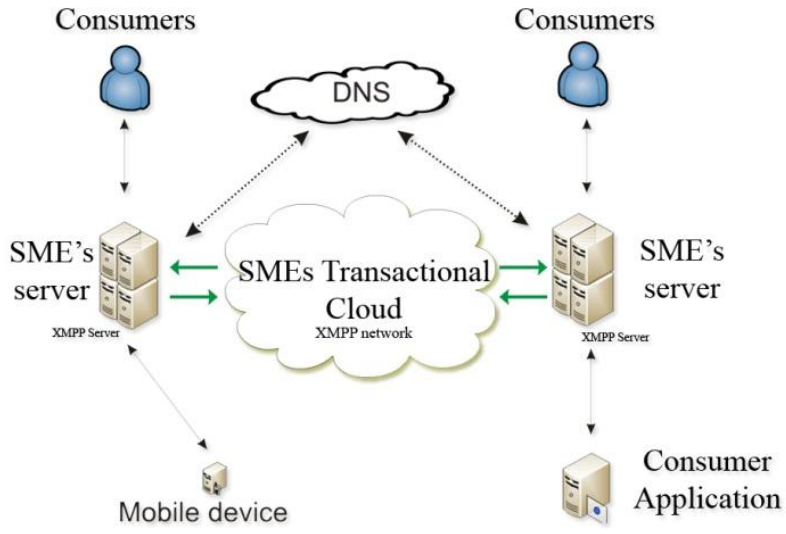

\section{Fig. 25 XMPP implementation for digital ecosystem}

Figure 25 outlines the general idea behind this integration which involves modelling XMPP servers onto customised SME's servers. In this case, the digital ecosystem infrastructure described in this paper can act as a SMEs transactional cloud, which dynamically optimises itself to respond to the usage that is being made of it based on the transactions taking place between participating organisations.

\section{CONCLUSIONS AND FUTURE WORK}

In this paper we have described the use of a P2P solution in the context of digital ecosystems, and in particular we have been concerned with services, transactions, and network support within the digital ecosystem initiative. The structure of the P2P network within the architecture we have proposed emerges through the local interactions that take place in the context of long-running business transactions. Particular care has been taken to ensure that transaction and P2P support in our approach satisfies certain requirements that are pertinent to the adoption of digital ecosystems by SMEs. The absence of a central point of command and control (and by virtue of that, also governance), and consequently the absence of a single point of failure, the distributed coordination, the usage of loosely-coupled services, the resilience to fragmentation and smart attacks, and allowing for a dynamic topology that continuously adapts to reflect the actual usage of the network in terms of business transactions are the main features that figure prominently in the proposed P2P network design.

In part, this has been achieved by considering a distributed model for the coordination of long-running transactions, and the provision for fine-grained lock schemes and recovery procedures. The transaction model feeds into the corresponding Virtual Private Transaction Networks (VPTNs) which are the main building block for the underlying P2P network that supports these complex interactions between participating entities.

The basic design feature of the P2P network has to do with the Virtual Super Peer (VSP) construct. These clusters of stable nodes are used instead of the conventional super peer solution and allow for creating a connected network without generating dependency on (a few or) a single network infrastructure provider. They are formed dynamically and this means the VSP solution also allows the network to reconfigure itself and withstand certain types of failure that typical scalefree networks find it difficult to recover from such as fragmentation or smart attacks.

Some of the basic features of the VPTNs go beyond the domain of business transactions and are relevant in more general complex interactions for example involving knowledge services. For this reason we have focused on correctness of the transaction model. In [19] we have been concerned with the order of execution, in terms of service invocations between various participants, aiming to prohibit undesirable behaviour while in this paper we have dealt with data-orientation by applying the lock scheme proposed in [35] and have shown that interactions can be done and undone in a consistent manner.

This is important not only because the VPTNs are the main building block for the underlying P2P network but for other applications over P2P networks. For instance, a prerequisite for online collaborative editing of documents (or content more generally) is that changes made by one participant are visible to the rest and not overwritten by their concurrent edits. The proposed lock scheme, here considered within the transaction model, allows for such simultaneous editing on different parts of the document, and this is something that is being incorporated in the guigoh e-learning collaborative platform in collaboration with IPTI as outlined in Section VI.

As mentioned before, a digital ecosystem is highly dynamic environment for a variety of reasons and failures of various types are to be expected. Therefore, our efforts so far have been targeted at providing a stable network that exhibits increased connectivity and resilience to fragmentation. For instance, time-zones and a pool of candidate nodes factor in the formation of VSPs and hence the proposed P2P network design works best as the number of nodes increases. Further experimentation is under way with regard to a number of parameters in the proposed framework such as the minimum number of nodes that afford desired stability levels, the period of time needed for forming layers of VSPs and this is in addition to other considerations such adapting efficient search algorithms into our framework. In terms of transactions, we are looking at other variable parameters such as the time-out in T-Lock and the overall execution period of a given transaction. Although these tend to be domain-specific, we are keen to exploit the interrelationship with the underlying network topology in providing a more stable environment for business activities and open collaboration.

Another factor that adds to the dynamicity of a digital 
ecosystem for business is that nodes (especially when considering SMEs) may be joining or leaving the network continuously, and in some cases abruptly. The proposed P2P network is essentially an unstructured network which only inherits from the VPTNs network structures, and in this paper we only touched upon the issues of birth and growth. In addition, the network topology itself evolves continuously based on the dynamically formed VSPs. In order to get a handle on how the network topology evolves under the events of nodes join and leaving the network, we have been looking at biological models of growth in living organisms. Of particular interest seems to be the study of molecular networks of lips and proteins in [36] which exhibit scale-free characteristics and have interesting properties with respect to connectivity. Preliminary investigations show that these aspects are driven by the major evolutionary events in growth in molecular networks, namely domain duplication and innovation. We are currently examining ways to inform the reaction of the network, possibly in terms of the neighbouring nodes, to the event of a node joining the network or leaving. This can be coupled with the component-based design of the local software agent on each participating node, and this is certainly an aspect of the work that we are keen to investigate further.

\section{ACKNOWLEDGMENT}

This work was supported by the EU-FP6 funded project OPAALS Contract No 034824.

\section{REFERENCES}

[1] Allee, V. Reconfiguring the Value Network. Journal of Business Strategy, 21(4), Jul-Aug 2000

[2] A. Razavi, S. Moschoyiannis and P. Krause. A Coordination Model for Distributed Transactions in Digital Business Ecosystems. In IEEE Int'l Conf. on Digital Ecosystems and Technologies (DEST'07), 2007.

[3] S. Moschoyiannis, A. Razavi, Y. Zheng and P. Krause. Long-running transactions: semantics, schemas, implementation. In IEEE Int'l Conf. on Digital Ecosystems and Technologies (DEST'08), 2008.

[4] A. Razavi, S. Moschoyiannis and P. Krause. A Scale-Free Business Network for Digital Ecosystems. In IEEE Int'l Conf. on Digital Ecosystems and Technologies (DEST'08), 2008.

[5] B. Y. Beverly and H. Garcia-Molina. Designing a super-peer network. In 19th Int'l Conf. on Data Engineering, pp. 49-60, 2003.

[6] B. Yang, H. Garcia-Molina. Improving search in peer-to-peer networks. In 22nd Int'l Conf. on Distributed Computing Systems, pp. 5-14, 2002.

[7] B. Martini, F. Baroncelli, P. Castoldi. A novel service oriented framework for automatically switched transport Network. In Integrated Network Management, pp. 295 - 308, 2005.

[8] Kristiansen L. et al. TINA Service Architecture and Specifications. TINA 1.0 Deliverables and Sepcification. Available at: http://www.tinac.com/specifications/specifications.htm

[9] Sahai A. et al. Automated SLA monitoring for web services. In 13th IFIP/IEEE International Workshop on Distributed Systems: Operations and Management: Management Technologies for E-Commerce and EBusiness Applications, p.28-41, 2002.

[10] Z. Li, P.Mohapatra, "QRON: QoS-Aware Routing in Overlay Networks", Service Overlay Networks in the IEEE Journal on Selected Areas in Communications (2004).
[11] Z.B. Daho, N. Simoni. Towards Dynamic Virtual Private Service Networks: Design and Self-Management. In 10th IEEE/IFIP Network Operations and Management Symposium (NCMS'06), 2006.

[12] F.H. Vogt, S. Zambrovski, B. Grushko et al. Implementing Web Service Protocols in SOA: WS-Coordination and WS-BusinessActivity. In Proc.7th IEEE Conf on E-Commerce Technology Workshops, pp. 21-26, IEEE Computer Society, 2005.

[13] P. Furnis and A. Green. Choreology Ltd. Contribution to the OASIS WS-TX Technical Committee relating to WS-Coordination, WSAtomicTransaction and WS-BusinessActivity. November 2005.

[14] A. Razavi, S. Moschoyiannis and P. J. Krause. "Preliminary Architecture for Autopoietic P2P Network focusing on Hierarchical Super-Peers, Birth and Growth Models." OPAALS project Deliverable D3.2, 2007 - available at: http://files.opaals.org/OPAALS

[15] http://fada.sourceforge.net/

[16] A. L Barabasi, R. Albert. Emergence of Scaling in Random Net-works. Science 286(5439): 509-512, 1999.

[17] Digital Business Ecosystems (DBE) EU IST Integrated Project No 507953. Available at http://www.digital-ecosystem.org .

[18] C. J. Date. An introduction to Database Systems. (8th edition) Addison Wesley, 2004.

[19] S. Moschoyiannis, A. Razavi. P. Krause. Transaction Scripts: making implicit scenarios explicit. In Proc. ETAPS 2008 - Formal Foundations of Embedded Software and Component-Based Architectures (FESCA'08), ENTCS, Elsevier, 2008. To appear

[20] M. Singh and M. N. Huhns. Service-Oriented Computing. Wiley Blackwell, 2004.

[21] P. Furnis, S. Dala, T. Fletcher et al. Business Transaction Protocol, version 1.1.0, November 2004. Available at http://www.oasisopen. org/committes/downaload.php [19 Sep 2006].

[22] P. Bernstein, N. Goodman, and V. Hadzilacos. Concurrency Control and Recovery in Database Systems. Addision-Welsley, 1987.

[23] J. Gray, A. Reuter. Transaction processing: Concepts and Techniques, Morgan Kaufmann Publishers, USA, 1993.

[24] L.F. Cabrera, G. Copeland, M. Feingold et al. Web Services Coordination (WS-Coordination). August 2005. Available http://www128.ibm.com/developerworks/webservices/library/specification/ws-tx [19 September 2006].

[25] A. Elmagarmid. Database Transaction Model for Advanced Applications, Morgan - Kaufmann, 1994.

[26] J. E. B. Moss. Nested transaction an approach to Reliable Distributed Computing. MIT Press, USA, 1985.

[27] L.F. Cabrera, G. Copeland, J. Johnson and D. Langworthy. Coordinating Web Services Activities with WS-Coordination, WSAtomicTransaction, and WS-BusinessActivity. January 2004. Available: http://msdn.microsoft.com/webservices/default.aspx [19 Sep 2006].

[28] M.S. Haghjoo, M.P. Papazoglou, "TrActorS: a transactional actor system for distributed query processing", Proceedings of the 12th International Conference on Distributed Computing Systems (IEEE CNF), pp: $682-689,1992$.

[29] L.F. Cabrera, G. Copeland, W. Cox et al. Web Services Business Activity Framework (WS-BusinessActivity). August 2005. Available http://www128.ibm.com/developerworks/webservices [19 Sep 2006]

[30] A. Razavi, P.J. Krause and S.K. Moschoyiannis. DBE Distributed Transaction Model. Digital Business Ecosystem (DBE) Report D24.28, University of Surrey, 2006.

[31] J. Yang, M. Papazoglou and W-J. van de Heuvel. Tackling the Challenges of Service Composition in E-Marketplaces. In Proc. 12th RIDE-2EC, pp. 125-133, IEEE Computer Society, 2002.

[32] M.P. Papazoglou. Service-Oriented Computing: Concepts, Characteristics and Directions. In Proc. WISE'03, IEEE, pp. 3-12, $2003 .$.

[33] http://www.opaals.org/

[34] http://personal.cs.surrey.ac.uk/personal/pg/A.Razavi/ppna/

[35] A. Razavi,S. Moschoyiannis,P. Krause: Concurrency Control and RecoveryManagement in Open e-Business Transactions. In: Proc. WoTUG Communicating Process Architectures (CPA 2007), pp. 267285. IOS Press, Amsterdam (2007)

[36] A. Rzhetsky, S. Gomez, "Birth of scale-free molecular networks and the number of distinct DNA and protein domains per genome", Bioinformatics, 17(10):988-996, 2001

[37] M. P. Papazoglou, P. Traverso, S. Dustdar, F. Leymann and B. J. Kramer. Service-Oriented computing Research Roadmap. In Dagstuhl Seminar Proceedings 05462, Service-Oriented Computing (SOC), pp. 129, 2006.

[38] Flypeer Opensource Project. http://kenai.com/projects/flypeer/ 